\title{
An Integrated Hardware-Software Approach to Flexible Transactional Memory*
}

\author{
Arrvindh Shriraman \\ Michael F. Spear \\ Hemayet Hossain \\ Virendra J. Marathe \\ Sandhya Dwarkadas \\ Michael L. Scott \\ Department of Computer Science, University of Rochester \\ $\{$ ashriram,spear,hossain,vmarathe,sandhya,scott $\} @$ cs.rochester.edu
}

TR $910 \dagger$

December 2006

\begin{abstract}
There has been considerable recent interest in the support of transactional memory (TM) in both hardware and software. We present an intermediate approach, in which hardware is used to accelerate a TM implementation controlled fundamentally by software. Our hardware support reduces the overhead of common TM tasks, namely, conflict detection and data isolation, for bounded transactions. Software control allows policy flexibility for conflict detection, contention management, and data granularity, in addition to enabling transactions unbounded in space and time. Our hardware consists of 1) an alert-on-update mechanism for fast eventbased communication, used for software-controlled conflict detection; and 2) support for programmable data isolation, allowing multiple concurrent transactional readers and writers at the software's behest, along with fast data commit and abort support (using only a few cycles of completely local operation).

Our results show that for common-case bounded transactions, the proposed hardware mechanisms eliminate data copying and dramatically reduce the overhead of bookkeeping and validation (resulting in a factor of 2 improvement in performance on average). Moreover, RTM shows good scalability as the number of threads is increased and graceful degradation in performance when transactions overflow available hardware support. Detecting conflicts eagerly (on first access) or lazily (at commit time), enabled by the ability to handle multiple concurrent transactional writers and readers, can result in differences in performance in either direction depending on the application access pattern (up to two orders of magnitude at 16 threads for one workload), demonstrating the need for policy flexibility.
\end{abstract}

${ }^{*}$ This work was supported in part by NSF grants CCR-0204344, CNS-0411127, CNS-0615139, and CNS-0509270; an IBM Faculty Partnership Award; financial and equipment support from Sun Microsystems Laboratories; and financial support from Intel and Microsoft.

${ }^{\dagger}$ Portions of this work were previously reported in URCS TR 887, December 2005, and in a paper at TRANSACT 2006 [32]. The current paper adds the RTM-Lite protocol, the simplified TMESI protocol (appendix B), additional detail and updates, and performance results. 


\section{Introduction and Background}

Explicitly parallel hardware, once confined to high-end servers and scientific computing, will soon dominate the full computing spectrum. As a result of increasing chip density coupled with power limitations, multicore chips - often chip multiprocessors (CMPs) of simultaneous multithreaded cores (SMTs) - are replacing uniprocessors throughout the desktop and laptop markets $[12,13,21]$. The range and scale of such multicore architectures is likely to increase, making support to ease the process of parallelizing applications imperative.

Transactional memory (TM) has emerged as a promising alternative to lock-based synchronization. TM systems seek to increase scalability, reduce programming complexity, and overcome the semantic problems of deadlock, priority inversion, and non-composability associated with locks. Originally proposed by Herlihy and Moss [10], TM borrows the notions of atomicity, consistency, and isolation from database transactions. In a nutshell, the programmer or compiler labels sections of code as atomic and relies on the underlying system to ensure that their execution is serializable and as highly concurrent as possible. Several pure hardware $[1,3,8,18,23,26-28]$ and software $[5,9,16,29,31]$ TMs have been proposed. Pure hardware TM proposals have the advantage of speed, but are typically highly ambitious and embed significant amounts of policy in silicon. Software TM proposals run on stock processors and provide substantial flexibility in policy, but incur significant overhead for data versioning and validation in the face of conflicting transactions.

Damron et al. [4] describe a design philosophy for a hybrid TM system in which hardware makes a "best effort" attempt to complete transactions, falling back to software when necessary. The goal is to leverage almost any reasonable hardware implementation. Kumar et al. [14] describe a specific hardwaresoftware hybrid that builds on the software system of Herlihy et al. [9]. Unfortunately, this system still embeds significant policy in silicon. It assumes, for example, that conflicts are detected as early as possible (pessimistic concurrency control), disallowing either read-write or write-write sharing. Scherer et al. [16,30] report performance differences across applications of $2 \times-10 \times$ in each direction for this design decision, and for contention management and metadata organization.

We propose that hardware serve simply to optimize the performance of transactions that are controlled fundamentally by software. We present a system, RTM, that embodies this philosophy. Our hardware support reduces the overhead of common TM tasks—conflict detection, validation, and data isolation-for common-case bounded transactions. Software control (currently based on a modified version of the RSTM software TM [17]) preserves policy flexibility and supports transactions unbounded in space and in time.

Our hardware consists of 1) an alert-on-update mechanism for fast software-controlled conflict detection; and 2) programmable data isolation, allowing potentially conflicting readers and writers to proceed concurrently under software control. Alert-on-update is the simpler and more general of the mechanisms. It can be used for any task that benefits from fine-grain access control. For TM, it eliminates the heavy cost of read-set validation [33]. Programmable data isolation additionally eliminates the cost of data copying or logging for bounded transactions.

By leaving policy to software, RTM allows us to experiment with a wide variety of policies, for conflict detection, contention management, deadlock and livelock avoidance, data granularity (e.g., word v. objectbased), nesting, and virtualization. We focus in this paper on conflict detection: We permit, but do not require, read-write and write-write sharing, with delayed detection of conflicts. We also employ a software contention manager [30] to arbitrate conflicts and determine the order of commits.

Because conflicts are handled in software, speculatively written data can be made visible at commit time with only a few cycles of entirely local execution. Moreover, these data (and a small amount of nonspeculative metadata) are all that must remain in the cache for fast-path execution: data that were speculatively read or nonspeculatively written can safely be evicted at any time. Like the Damron and Kumar hybrid 
proposals, RTM falls back to a software-only implementation of transactions in the event of overflow, but in contrast not only to the hybrid proposals, but also to TLR [27], LTM [1], VTM [28], and LogTM [23], it can accommodate "fast path" execution of dramatically larger transactions with a given size of cache. Nonspeculative loads and stores are permitted in the middle of transactions-in fact they constitute the hook that allows us to implement policy in software.

The RTM hardware described in this paper is intended for implementation either at the L1 level of a CMP with a shared L2 cache, or at the L2 level of an SMP with write-through L1 caches. We describe an implementation based on the classic MESI broadcast protocol. Other implementations (for directory-based protocols) are a subject of ongoing work. Likewise, while our current software inherits a variety of policies (in particular, nonblocking semantics and object-level granularity) from RSTM, our hardware could be used with a variety of other software TMs, including systems that track conflicts at word granularity or use locks to make updates in place.

For a suite of microbenchmarks with varying access patterns, we find that RTM improves the performance of common-case bounded transactions by as much as a factor of two. Moreover, RTM shows good scalability as the number of threads increases. The proposed hardware mechanisms eliminate data copying and dramatically reduce the overhead of bookkeeping and validation. RTM-Lite, a simpler system that relies on alert-on-update but not programmable data isolation, is effective at eliminating validation overhead, but loses to RTM for transactions that modify large objects. When transactions overflow the available hardware support, performance degrades linearly from that of the all fast-path mode to that of the all overflow mode, based on the fraction of overflowed transactions. Detecting conflicts eagerly (on first access) or lazily (at commit time), enabled by the multiple-writer protocol, can result in differences in performance in either direction depending on the application access pattern (up to two orders of magnitude for one workload), demonstrating the need for policy flexibility.

Section 2 describes our hardware mechanisms in detail, including instruction set extensions, coherence protocol, and the mechanism used to detect conflicts and abort remote transactions. Section 3 then describes the RTM runtime that leverages this hardware support. Section 4 evaluates the performance of RTM in comparison to coarse-grain locks, an all-software TM system, and an "RTM-Lite" system that uses alert-onupdate but not programmable data isolation. Section 4 also presents results to demonstrate the benefits of policy flexibility. We conclude in Section 5 with a summary of contributions and a list of topics for future research.

\section{Hardware Support}

Transactional memory has two principal requirements: the ability to detect when transactions in different threads conflict, and the ability to hide a transaction's updates from other threads until the transaction commits. These requirements can be met in hardware or in software. We describe hardware mechanismsalert-on-update and programmable data isolation - that can accelerate software implementations without constraining policy.

\subsection{Alert-On-Update}

To enable conflict detection, we propose a simple technique to selectively expose coherence events (potential writes by other processors) to user programs: threads register an alert handler and then selectively mark lines of interest as alert-on-update (AOU). When a cache controller detects a remote write or eviction of a line that is marked in the local cache, it notifies the local processor, effecting a spontaneous subroutine call to the current thread's alert handler. Because the state of a line may change due to conflict or capacity misses on other lines, a handler must in general double-check the cause of the alert. 


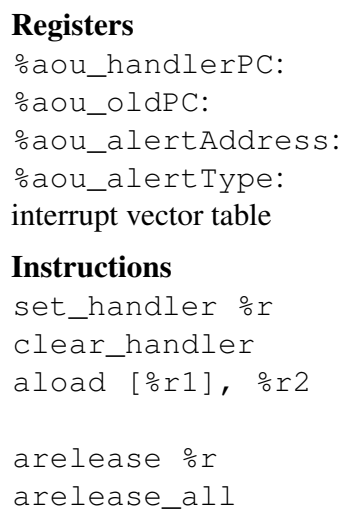

Table 1: Alert-on-update hardware requirements. address of the handler to be called on a user-space alert program counter immediately prior to the call to $\%$ aou_handlerPC address of the line whose status change caused the alert remote_write, lost_alert, or capacity/conflict eviction one extra entry to hold the address of the handler for kernel-mode alerts

move $\%$ into $\%$ aou_handlerPC clear $\circ$ aou_handlerPC and flash-clear the alert bits for all cache lines load the word at address $\circ r 1$ into register $\circ r 2$, and set the alert bit(s) for the corresponding cache line unset the alert bit for the cache line that corresponds to the address in register $\circ \mathrm{r}$ flash-clear alert bits on all cache lines

Implementation Implementation of alert-on-update relies on the cache coherence protocol, but is essentially independent of protocol details. Coherence requires, by definition, that a controller be notified when the data cached in a local line is written elsewhere in the machine. The controller also knows of conflict and capacity evictions. We simply alert the processor of these events when they occur on lines that have previously been marked. The alert includes the address of the affected line and the nature of the event.

Table 1 summarizes hardware requirements. These include special registers to hold the address of the user-mode handler and a description of the current alert; an extra entry in the interrupt vector table (for alerts that happen while running in kernel mode); and instructions to set and unset the user-mode handler and to mark and unmark cache lines (i.e., to set and clear their alert bits). The marking instruction, aload, also returns a word of the line.

ALoads serve two related roles in RTM, which we describe in more detail in Section 3. First, every transaction ALoads a word that describes its current status. If any other transaction aborts it (by modifying this word), the first transaction is guaranteed to notice. Second, a transaction can ALoad a word of metadata associated with a given object. If writers modify that word before committing changes to the object, readers are guaranteed to notice. (Significantly, this mechanism does not require that conflict be detected as soon as some word of the object is speculatively written, thereby permitting lazy conflict detection.)

\subsection{Programmable Data Isolation}

Caches inherently provide data buffering, but coherence protocols normally propagate modifications quickly to all copies. As in most hardware transactional memory proposals $[1,8,23,28]$, we allow a thread to delay this propagation while executing speculatively, and then to make an entire set of changes visible to other threads atomically. We use a level of cache close to the processor to hold the new copy of data, and rely on shared lower levels of the memory hierarchy to hold the old values of lines. Unlike most other hardware TM designers, however, we allow lines to be read and written transactionally even when they are also being written by some other, concurrent transaction.

Implementation We describe an implementation based on the traditional MESI coherence protocol, which we label TMESI. Table 2 summarizes hardware requirements.

Potentially speculative reads and writes use TLoad and TStore instructions. These instructions are interpreted as speculative when the transactional bit (\%t_in_flight) is set. As described in Section 3, this allows the same code path to be used by both fast-path transactions and those that overflow the available 


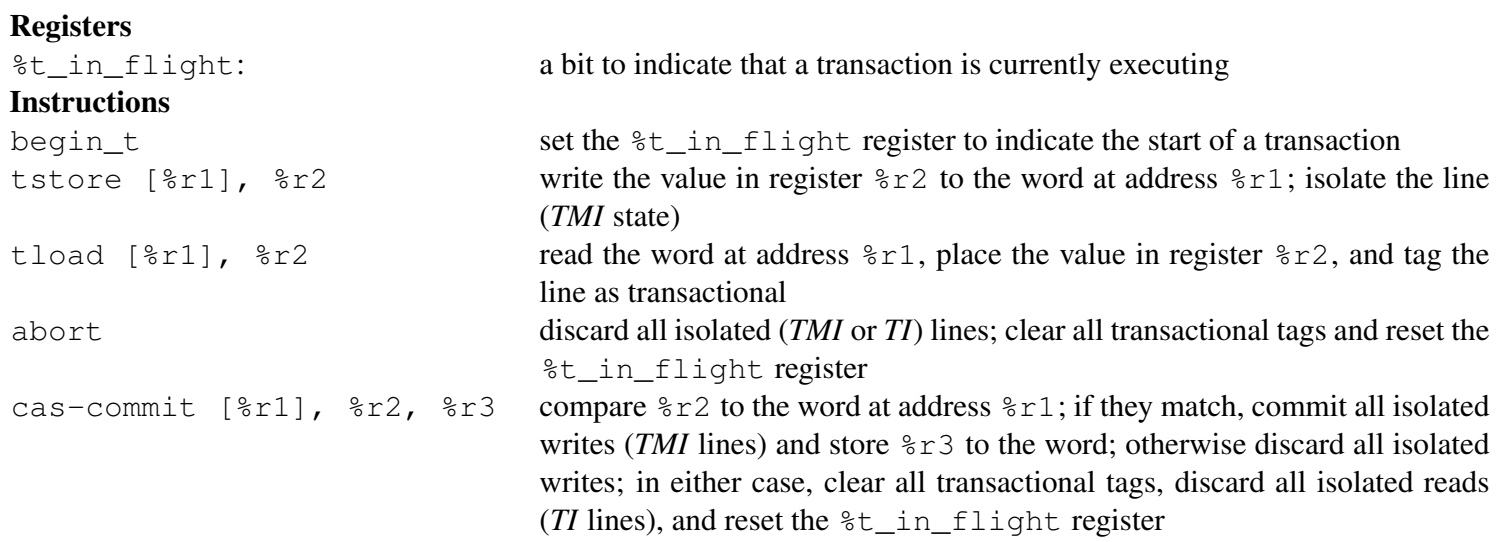

Cache

two extra stable states, TMI and TI, for isolated reads and writes;

transactional tag for the MES states

Table 2: TMESI hardware requirements.

hardware support. TStore is used for writes that require isolation. TLoad is used for reads that can safely be cached despite remote TStores.

Speculative reads and writes employ two new coherence states: TI and TMI. These states allow a software policy, if it chooses, to perform lazy detection of read-write and write-write conflicts. Hardware helps in the detection task by piggybacking a threatened $(\mathrm{T})$ signal/message, analogous to the traditional shared (S) signal/message, on responses to read-shared bus requests whenever the line exists in TMI state somewhere in the system. The $\mathrm{T}$ signal warns a reader of the existence of a potentially conflicting writer.

$T M I$ serves to buffer speculative local writes. Regardless of previous state, a line moves to TMI in response to a PrTWr (the result of a TStore). A TMI line then reverts to $M$ on commit and to $I$ on abort. Software must ensure that among any concurrent conflicting writers, at most one commits, and if a conflicting reader and writer both commit, the reader does so first from the point of view of program semantics. The first TStore to a modified cache line results in a writeback prior to transitioning to TMI to ensure that lower levels of the memory hierarchy have the latest non-speculative value. A line in TMI state threatens read requests and suppresses its data response, allowing lower levels of the hierarchy to supply the non-speculative version of the data.

TI allows continued use of data that have been read by the current transaction, but that may have been speculatively written by a concurrent transaction in another thread. An $I$ line moves to $T I$ when threatened during a TLoad; an $M, E$, or $S$ line moves to $T I$ when written by another processor while tagged transactional (indicating that a TLoad has been performed by the current transaction). A TI line must revert to $I$ when the current transaction commits or aborts, because a remote processor has made speculative changes which, if committed, would render the local copy stale. No writeback or flush is required since the line is not dirty. Even during a transaction, silent eviction and re-read is not a problem because software ensures that no writer can commit unless it first aborts the reader.

The CAS-Commit instruction performs the usual function of compare-and-swap. In addition, if the CAS succeeds, speculatively written (TMI) lines revert to $M$, thereby making the data visible to other readers through normal coherence actions. If the CAS fails, TMI lines are invalidated, and software branches to an abort handler. In either case, speculatively read $(T I)$ lines revert to $I$ and any transactional tags are flashed clear on $M, E$, and $S$ lines. The motivation behind $C A S$-Commit is simple: software TM systems invariably use a CAS or its equivalent to commit the current transaction; we overload this instruction to make buffered transactional state once again visible to the coherence protocol. The Abort instruction clears the transactional 


\begin{tabular}{|c|c|c|c|c|c|c|c|}
\hline $\mathrm{T}$ & A & MESI & $\mathrm{C} / \mathrm{A}$ & $\mathrm{M} / \mathrm{I}$ & State & \multirow{12}{*}{$\begin{array}{l} \\
\mathrm{T} \\
\mathrm{A} \\
\mathrm{MESI} \\
\mathrm{C} / \mathrm{A} \\
\mathrm{M} / \mathrm{I}\end{array}$} & \multirow{12}{*}{$\begin{array}{l}\text { Line is }(1) / \text { is not }(0) \text { transactional } \\
\text { Line is ( } 1) / \text { is not }(0) \text { alert-on-update } \\
2 \text { bits: I }(00), \mathrm{S}(01), \mathrm{E}(10) \text {, or M }(11) \\
\text { Most recent txn committed (1) or aborted ( } 0 \\
\text { Line is/was in TMI (1) }\end{array}$} \\
\hline 0 & - & 00 & - & - & $\lambda_{\mathrm{I}}$ & & \\
\hline 0 & - & 11 & 0 & 1 & \}$^{1}$ & & \\
\hline 0 & - & 01 & - & - & $\mathrm{S}$ & & \\
\hline 0 & - & 10 & - & - & $\mathrm{E}$ & & \\
\hline 0 & - & 11 & 1 & - & $\mathrm{M}$ & & \\
\hline 0 & - & 11 & 0 & 0 & & & \\
\hline 1 & - & 00 & - & - & TI & & \\
\hline 1 & - & 01 & - & - & $\mathrm{TS}$ & & \\
\hline 1 & - & 10 & - & - & $\mathrm{TE}$ & & \\
\hline 1 & - & 11 & - & 0 & $\mathrm{TM}$ & & \\
\hline 1 & - & 11 & - & 1 & TMI & & \\
\hline
\end{tabular}

Table 3: Tag array encoding for fast commits and aborts. The A (alert) bit is orthogonal to the above states.

state in the cache in the same manner as a failed CAS-Commit.

To the best of our knowledge, RTM and TCC [8] are the only hardware or hybrid TM systems that support read-write and write-write sharing; other schemes all perform eager conflict detection at the point where a conventional coherence protocol must invalidate a speculatively read line or demote a speculatively written line. By allowing a reader transaction to commit before a conflicting writer, RTM permits significant concurrency in the face of long-running writers. Write-write sharing is more problematic, since it can't result in more than one commit. Even so, it allows us to avoid aborting a transaction in favor of a competitor that is ultimately unable to commit; it may also be desirable in conjunction with early release [16]. Note that nothing about the TMESI protocol requires read-write or write-write sharing; if the software protocol detects and resolves conflicts eagerly, the $T I$ state will simply go unused.

To simplify the management of metadata, our implementation of RTM employs a Wide-CAS instruction (not shown in Table 2) that implements compare-and-swap across multiple contiguous locations (within a single cache line). As in Itanium's cmp $8 \mathrm{xchg} 16$ instruction [11], if the first two words at location A match their "old" values, all words are swapped with the "new" values (loaded into contiguous registers). Success is detected by comparing old and new values in the registers.

\subsection{Cache tag encoding}

All told, a TMESI cache line can be in any one of the four MESI states $(I, S, E, M)$, the two speculative states $(T I, T M I)$, or transactionally tagged variants of $M, E$, and $S$. If the protocol were implemented as a pure automaton, this would imply a total of 9 stable states, compared to 4 in the base protocol. Regular MESI also has three transient states, which the automaton enters while waiting for a response to a remote message. TMESI adds four additional such states, for a total of 16 rather than 7 . Any of these states could also be tagged as alert-on-update.

It would be possible to eliminate the transactionally tagged $M E S$ states entirely, at the cost of some extra reloads in the event of read-write sharing. Suppose thread $T 1$ has read line $X$ transactionally at some point in the past. The transactional tag indicates that $X$ was TLoaded as part of the current transaction. A remote write to $X$ (appearing as a BusRdX protocol message) can move $X$ to $T I$, because software will be tracking potential conflicts, and will allow $T 1$ to commit only if it does so before any conflicting transaction commits. If TLoads are replaced with normal loads and/or the transactional tags eliminated, $T 1$ will need to drop $X$ to $I$, but a subsequent load will bring it back to $T I$. Evaluating the tradeoff between complexity and reloads is a subject of future work.

To allow fast commits and aborts, our cache tags can be encoded in six bits, as shown in Table 3. At commit time, based on the outcome of the CAS in CAS-Commit, we broadcast a 1 (or 0) on the C/A bit line 
and use the $T$ bits to conditionally enable only the tags of transactional lines. Following this, we flash-clear the $\mathrm{A}$ and $\mathrm{T}$ bits. For $T M, T E, T S$, and $T I$ the flash clear alone would suffice, but $T M I$ lines must revert to $M$ on commit and $I$ on abort. We use the C/A bit to distinguish between these: when the line is next accessed, $\mathrm{M} / \mathrm{I}$ and $\mathrm{C} / \mathrm{A}$ are used to interpret the state before being reset. If $\mathrm{T}$ is 0 , the $\mathrm{MESI}$ bits are $11, \mathrm{C} / \mathrm{A}$ is 0 , and $\mathrm{M} / \mathrm{I}$ is 1 , the cache line state is invalid and the MESI bits are changed to reflect this. In all other cases, the state reflected by the MESI bits is correct.

\section{RTM Runtime}

The RTM runtime is based on the open-source RSTM system [17], a C++ library that runs on legacy hardware. RTM uses alert-on-update and programmable data isolation to avoid copying and to reduce bookkeeping and validation overheads, thereby improving the speed of "fast path" transactions. When a transaction's execution time exceeds a single quantum, or when the working set of a transaction exceeds the ALoad or TStore capacity of the cache, RTM restarts the transaction in a more conservative "overflow mode" that supports unboundedness in both space and time.

\subsection{The RTM API}

RTM is $100 \%$ source-code compatible with RSTM. Details of the API can be found elsewhere [17]. Briefly, transactions are lexically scoped, and delimited by BEGIN_TRANSACTION and END_TRANSACTION macros. BEGIN_TRANSACTION sets the alert handler for a transaction and configures per-transaction metadata. END_TRANSACTION issues a CAS-Commit.

Objects accessed transactionally must derive from a provided generic transaction class. Among other things, the transactional object provides access to transaction-safe memory management routines. (We can use any thread-safe memory allocator, and indeed we have experimented with many. The transactional object wraps any given allocator in code that defers the reuse of deleted space until we are certain that no doomed transaction retains a stale pointer to the object.)

In order to access fields of the object, the programmer must request read or write permission by performing an open_RO or open_RW call, which returns a pointer to the object. These calls are explicit in the source code, though with compiler support they could easily be generated automatically. They fail if performed outside a transaction. The interface also provides a release method [9], which allows a programmer with application-specific semantic knowledge to inform the runtime that conflicts on the object are no longer cause to abort the transaction. Release is a potentially unsafe optimization, which must be used with care.

One additional operation, used primarily inside the run-time system, is also available to programmers. The runtime often requires that a set of related metadata updates be allowed to complete, i.e., that the transaction not be aborted immediately. This is accomplished by using a flag to indicate that the set of updates are in progress. If an alert occurs while the flag is set, the handler defers its normal actions, sets another flag, and returns. When the runtime finishes its updates, it clears the first flag, checks the second, and jumps back to the handler if action was deferred. Applications can use this "deferred abort" mechanism to protect a set of related updates to nontransactional data from within a transaction (e.g., for logging purposes). In effect, deferred abort blocks serve as a cheap, non-isolated approximation of open nested transactions [24].

\subsection{Metadata}

Every RTM transaction is represented by a descriptor (Figure 1) containing a serial number and a word that indicates whether the transaction is currently ACTIVE, COMMITTED, or ABORTED. The serial number is incremented every time a new transaction begins. It enables the reuse of descriptors without the need for 


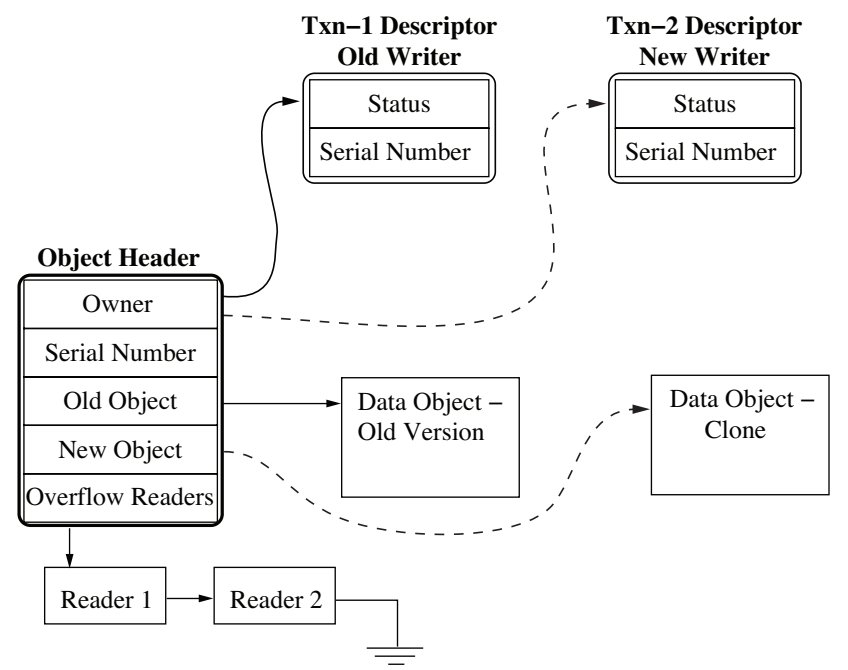

Here a writer transaction is in the process of acquiring the object, overwriting the Owner pointer and Serial Number fields, and updating the Old Object pointer to refer to the previously valid copy of the data. A fast-path transaction will set the New Object field to null; an overflow transaction will set it to refer to a newly created clone. Several overflow transactions can work concurrently on their own object clones prior to acquire time, just as fast-path transactions can work concurrently on copies buffered in their caches.

Figure 1: RTM metadata structure.

cleanup in the wake of a successful commit. If the serial numbers of an object header and descriptor do not match, then the descriptor's status is assumed to be COMMITTED. ABORTED overflow transactions must clean up all references to their descriptors before they start a new transaction.

Every transactional object is represented by a header containing five main fields: a pointer to an "owner" transaction, the owner's serial number, pointers to valid (old) and speculative $(n e w)$ versions of the object, and a head pointer to a list of overflow transactions currently reading the object.

The open_RO method returns a pointer to the most recently committed version of the object. Typically the owner/serial number pair indicates a COMMITTED transaction, in which case the New pointer is valid if it is not NULL, and otherwise the Old pointer is valid. If the owner/serial number pair indicates an ABORTED transaction, then the old pointer is always valid. When the owner is ACTIVE, there is a conflict. An object never has entries in the overflow readers list while there is an ACTIVE owner.

The open_RW method returns a pointer to a writable copy of the object. For fast-path transactions this is the valid version that would be returned by open_RO; updates will be buffered in the cache. For overflow transactions it is a clone or copy of the valid version.

At some point between its open_RW and commit time, a transaction must acquire every object it has written. The acquire operation first gets permission from a software contention manager $[9,30]$ to abort all transactions in the overflow reader list. It then writes the owner's ID, the owner's serial number, and the addresses of both the last valid version and the new speculative version into the header using (in our current implementation) a Wide-CAS instruction. Finally, it aborts any transactions in the overflow reader list of the freshly acquired object. ${ }^{1}$

At the end of a transaction, a thread issues a CAS-Commit to change its state from ACT IVE to COMMITTED (or ABORTED, in which case the transaction can be retried).

\subsection{Policy Flexibility}

RTM uses software to detect and resolve transaction conflicts. Two transactions conflict only if they access the same object and at least one of them attempts to write it. In RTM this conflict is not visible until the writer acquires the object. Under eager conflict detection, acquisition occurs at open time, and read-write and

\footnotetext{
${ }^{1}$ It is possible for a reader to enter the list after the acquirer finishes requesting permission to abort readers. In such circumstances, the late-arriving reader will be aborted without arbitration, which ensures correctness though not fairness.
} 
write-write sharing are precluded. A writer aborts any extant readers, and once there is a writer, subsequent readers and writers must abort the eager writer before they can access the object. In contrast, under lazy conflict detection, acquisition is deferred until commit time, and read-write and write-write sharing are permitted. Eager acquisition results in less overall bookkeeping since it doesn't require a final acquire phase, making it faster for workloads in which aborts are rare. It may also avoid useless work by aborting a doomed transaction early. Lazy acquisition, by contrast, avoids the possibility of aborting a transaction in favor of a competitor that is subsequently unable to commit. It also allows a writer and one or more concurrent readers to all commit, so long as the readers do so first.

When conflicts are detected, RTM uses a software contention manager to decide which transaction should continue, and which should wait or abort. Contention management policies are the subject of much ongoing research $[6,30]$; we currently support eight common policies, as well as others of our own design.

In a typical hardware TM system (e.g., LogTM [23]), contention management is embedded in the cache coherence protocol. It is performed by the controller that owns the line, based on the limited information available to that controller, while the requesting process blocks waiting for a response. In RTM, contention management is performed by nonblocking software, executed by the thread that discovers the conflict, using whatever information the runtime designer deems useful.

\subsection{Fast-Path RTM Transactions}

Eliminating Data Copying A fast-path transaction calls beg in_t inside the BEGIN_TRANSACTION macro. Subsequent TStores will be buffered in the cache, and will remain invisible to other threads until the transaction commits. As noted in Section 3.2, open_RW returns a pointer to the current version of an object when invoked by a fast-path transaction, thereby enabling in-place updates. Programmable data isolation thus avoids the need to create a separate writable copy, as is common in software TM systems (RSTM among them). When a fast-path transaction acquires an object, it writes a NULL into the New pointer, since the old pointer is both the last and next valid version. As a result, when a fast-path transaction aborts, it does not need to clean up the Owner pointers in objects it has acquired; because the owner has been working directly on the Old version of the data, a newly arriving transaction that sees mis-matched serial numbers will read the appropriate version.

Reducing Bookkeeping and Validation Costs In most software TM systems, a transaction may be doomed to fail (because of conflicting operations in committed peers) well before it notices its fate. In the interim it may read versions of objects that are mutually inconsistent. This possibility raises the problem of validation: a transaction must ensure that inconsistent data never cause it, erroneously, to perform operations that cannot be rolled back. In general, a transaction must verify that all its previously read objects are still valid before it performs any dangerous operation. The resulting overhead can be a major component of the cost of software TM [33]: making readers visible to writers requires metadata updates that induce large numbers of cache misses; leaving them invisible leads to $O\left(n^{2}\right)$ total cost for a transaction that reads $n$ objects.

ALoad allows validation to be achieved essentially for free. Whenever an object is read (or opened for writing with lazy acquire), the transaction uses ALoad to mark the object's header in the local cache. Since transactions cannot commit changes to an object without modifying the object header first, the remote acquisition of a locally ALoaded line results in an immediate alert to the reader transaction. Since the header must be read in any case, the ALoad induces no extra overhead. Freed of the need to explicitly validate previously opened objects, software can also avoid the bookkeeping overhead of maintaining those objects on a list. Best of all, perhaps, a transaction that acquires an object implicitly aborts all fast-path readers of that object simply by writing the header: fast-path readers need not add themselves to the list of readers in the header, and the $O(t)$ cost of aborting the readers is replaced by the broadcast invalidation 
already present in the cache coherence protocol.

One extra complication arises from programmable data isolation: since committing a fast-path writer updates written objects in-place, we must ensure that a transaction in overflow mode also notices immediately when it is aborted by a competitor. We therefore require that every transaction ALoad its own descriptor. If a competitor CAS-es its status to ABORTED, the transaction will suffer an alert, avoiding the possibility that it will read mutually inconsistent data from within a single object.

\subsection{Overflow RTM Transactions}

Like most hardware TM proposals, fast-path RTM transactions are bounded by space and time constraints. They cannot ALoad or TStore more lines than the cache can hold, and they cannot execute across a context switch, because we do not (currently) associate transaction IDs with tagged lines in the cache. To accommodate transactions that exceed these time and space bounds, RTM provides an overflow mode with only one hardware requirement: that the transaction's ALoaded descriptor remain in the cache whenever the transaction is running.

Disabling Speculative Loads and Stores In principle, a transaction that exceeds the capacity of the cache could continue to use the available space for as many objects as fit. For the sake of simplicity we do not currently pursue this possibility. Rather, a transaction that suffers a "no more space" alert aborts and retries in overflow mode. In this mode it leaves the $\circ$ t_in_flight bit clear, instructing the hardware to interpret TLoad and TStore instructions as ordinary loads and stores. This convention allows the overflow transaction to execute the exact same code as fast-path transactions; there is no need for a separate version.

Without speculative stores, however, the overflow transaction must clone objects it intends to write. When objects are acquired, the WCAS instruction writes the address of the clone into the New field of the metadata. When transactions encounter a header whose last Owner is committed and whose New field is non-null, they return the New version as the current valid version.

Limiting ALoads Since overflow transactions can run concurrently with fast-path transactions, they must abort whenever objects they are reading are overwritten. Though an overflow transaction cannot ALoad every object header it reads, it still ALoads its own descriptor. It also writes itself into the Overflow Reader list of every object it reads; this ensures it will be explicitly aborted by any writers on those objects.

Cloning and Consistency While only one ALoaded line is necessary to ensure immediate aborts and to handle validation, using a second ALoad can improve performance when a fast-path transaction and an overflow transaction are concurrent writers. If the overflow writer is cloning an object when the fast-path writer commits, the clone operation may return an internally inconsistent object. If the overflow transaction becomes a visible reader first, the problem is avoided. It is simpler, however, to ALoad the header and then clone the object. If another transaction commits changes, the clone operation will suffer an alert. We assume in our experiments that the hardware is able (with a small victim cache) to prefer non-ALoaded lines for eviction, and to keep at least two in the cache.

Context Switches To support transactions that must be preempted, we require two actions from the operating system. When it swaps a transaction out, the operating system flash clears all the $A$ tags. In addition, for transactions in fast-path mode, it executes the abort instruction to discard isolated lines. When it swaps the transaction back in, it starts execution in a software-specified restart handler (separate from the alert handler). The restart handler aborts and retries if the transaction was in fast-path mode or was swapped out in mid-clone; otherwise it re-ALoads the transaction descriptor and checks that the transaction

status has not been changed to ABORTED. If this check succeeds, control returns as normal; otherwise the transaction jumps to its abort code. 


\subsection{RTM-Lite}

While both alert-on-update and programmable data isolation can improve the performance of TM, alerton-update is a much smaller change to existing cache designs-an ALoad-capable processor can, in fact, be pin compatible with existing hardware. An analysis of overheads in software TM also suggested that alert-on-update alone could yield significant performance gains. We therefore designed a system that relies on this mechanism only.

Like a fast-path RTM transaction, an RTM-Lite transaction ALoads the headers of the objects it reads. It does not add itself to Overflow Reader lists. Since TStore is not available, however, it must clone every acquired object. At the same time, it never has to worry about in-place updates, so immediate aborts are not required. This avoids some complexity in the run-time system: the alert handler simply sets the descriptor to ABORTED and returns. A transaction checks its status on every API call, but this takes constant time: in comparison to RSTM, validation requires neither a cache-miss-inducing change to a visible reader list nor an $O(n)$ check of $n$ previously-opened objects.

As it turns out, RTM-Lite transactions resemble RSTM transactions more closely than they resemble either fast-path or overflow transactions in RTM. In recognition of this fact, we created the RTM-Lite code base by adding ALoads to RSTM and removing validation, rather than by removing in-place update from RTM. As a result, RTM-Lite shares some additional, incidental similarities to RSTM: Instead of using a Wide-CAS to update multiple header fields atomically, RTM-Lite moves several fields into the data object and requires an extra level of indirection to read an object whose owner has aborted. Instead of using serial numbers to recognize re-used descriptors, RTM-Lite requires both committed and aborted transactions to clean up Owner pointers in acquired objects.

Every RTM-Lite transaction keeps an estimate of the number of lines it can safely ALoad. If it opens more objects than this, it keeps a list of the extra objects and validates them incrementally, as RSTM does. If it suffers a "no more space" alert, it reduces its space estimate, aborts, and restarts. On a context switch, RTM-Lite transactions abort and restart as RSTM transactions.

\section{Evaluation}

In this section we present experimental results to evaluate our three main claims: that the RTM hardware can be effectively used to speed a software TM system, that policy flexibility is important, and that our hybrid design permits a heterogeneous mix of fast-path and overflow transactions without impeding throughput.

\subsection{Evaluation Framework}

We evaluate RTM through full system simulation of a 16-way chip multiprocessor (CMP) with private split L1 caches and a shared L2. We use the GEMS/Simics infrastructure [19], a full system functional simulator that faithfully models the SPARC architecture. The instructions specified in Section 2 are called through the standard Simics "magic instruction" mechanism. We implemented the TMESI protocol and alert-on-update mechanism using the SLICC [19] framework to encode all the stable and transient states in the system.

We employ GEMS's network model for bus and switch contention, using the parameters in Table 4. Simics allows us to run an unmodified Solaris 9 kernel on our target system with the "user-mode-change" and "exception-handler" interface enabling us to trap user-kernel mode crossings. On crossings, we suspend the current transaction context and allow the OS to handle TLB misses, register-window overflow, and other kernel activities required by an active user context in the midst of a transaction. On transfer back from the kernel we deliver any alert signals received during the kernel routine, triggering the alert handler as needed. On context switches, we simulate the execution of the simple software handlers described in Section 3.5. 


\begin{tabular}{|c|l|}
\hline \multicolumn{2}{|c|}{ 16-way CMP, Private L1, Shared L2 } \\
\hline Processor Cores & 16 1.2GHz in-order, single issue, ideal IPC=1 \\
\hline Private L1 Cache & 64kB 4-way split, 64-byte blocks, 1 cycle latency, 32-entry victim buffer \\
\hline Shared L2 Cache & 8MB, 8-way unified, 64-byte blocks, 4 banks, 20 cycle latency \\
\hline Memory & 2GB, 100 cycle latency \\
\hline Interconnection Network & 4-ary totally ordered hierarchical tree, 1 cycle link latency, 64-byte links \\
\hline
\end{tabular}

Table 4: Target System Parameters

\begin{abstract}
HashTable: Transactions use a hash table with 256 buckets and overflow chains to lookup, insert, or delete a value in the range $0 \ldots 255$ with equal probability. At steady state, the table is $50 \%$ full.

RBTree: In the red-black tree (RBTree) benchmark, transactions attempt to insert, remove, or delete values in the range $0 \ldots 4095$ with equal probability. At steady state there are about 2048 objects, with about half of the values stored in leaves.

RBTree-Large: This version of the RBTree benchmark uses 256-byte tree nodes to increase copying overheads. Transactions only modify a small percent of the fields of the node.

LFUCache: LFUCache uses a large (2048) array based index and a smaller (255 entry) priority queue to track the most frequently accessed pages in a simulated web cache. When re-heapifying the queue, transactions always swap a value-one node with a value-one child; this induces hysteresis and gives each page a chance to accumulate cache hits. Pages to be accessed are randomly chosen using a Zipf distribution: $p(i) \propto \Sigma_{0<j \leq i} j^{-2}$.

LinkedList-Release: In the LinkedList-Release benchmark, early release is used to minimize read-set size while performing inserts, lookups, and deletes into a sorted, singly-linked list holding values in the range $0 \ldots 255$.

RandomGraph The RandomGraph benchmark requires transactions to insert or delete vertices from an undirected graph represented with adjacency lists. Edges in the graph are chosen at random, with each new vertex initially having up to 4 randomly selected neighbors.
\end{abstract}

Table 5: Workload Description

We consider the six benchmarks listed in Figure 5, designed to stress different aspects of software TM. In all benchmarks, we execute a fixed number of transactions in single-thread mode to ensure that the data structure is in a steady state. We then instruct each thread to execute a fixed number of transactions concurrently, to evaluate throughput and scalability.

\title{
4.2 Runtime Systems Evaluated
}

We evaluate each benchmark with two RTM configurations. RTM-F always executes fast-path transactions to extract maximum benefit from the hardware; RTM-O always executes overflow transactions to demonstrate worst-case throughput. We also compare RTM to the freely-available RSTM [17] package and the RTM-Lite runtime described in Section 3.6, which uses ALoad to optimize the RSTM library. As a baseline we compare against a coarse-grain locking library (CGL), which enforces mutual exclusion by mapping the BEGIN- and END-TRANSACTION macros to acquisition and release of a single coarse-grain test-andtest-and-set lock.

To ensure a fair comparison, we use the same benchmark code, memory manager, and contention managers in all systems. For contention management we use the Polka manager [30] unless otherwise specified. The only exception to our code reuse is for simulating TLoad and TStore without compiler support. We modified the allocator to segregate the heap and construct all shared object payloads in high address ranges. Memory management proceeds as in all other systems, but our simulator treats all memory operations on high addresses as tloads and tstores. 


\subsection{Throughput and Latency}

Figure 2 presents the normalized throughput (transactions per second) for all benchmark and runtime combinations. We only consider eager transactions, and results are normalized to single-thread CGL performance, except for RandomGraph, which is normalized to single-thread RSTM performance. RTM-F, RTM-O, RTM-Lite, and RSTM demonstrate similar scaling behavior across all benchmarks; RTM-F and RTM-Lite appear to successfully leverage our hardware to achieve up to a $\sim 5 \times$ speedup at one thread, and consistently better performance at all thread levels, in comparison to RSTM.

To gain further insight into the improvements of RTM and RTM-Lite over RSTM, Figure 3 presents a breakdown of single thread execution time. The segments of each bar reflect time spent in various portions of user and API code. Real Work expresses time in user-provided code between the BEGIN- and END-TRANSACTION macros (time in user code outside of these macros is Non-Tx). Validation records any time spent by the runtime explicitly validating its read set, and Copy reflects time spent making clones of objects. MM records time spent in memory management. Since the individual metadata manipulations and bookkeeping operations occur at a very fine granularity, we include all time for these operations in Bookkeeping. For these single-thread runs, no time is spent on aborted transactions or contention management.

As the breakdowns show, RTM and RTM-Lite appear to successfully leverage ALoad to eliminate RSTM's validation overhead without a significant increase in bookkeeping. RTM is also able to eliminate the copying costs. Due to the small object sizes for most of the benchmarks (other than RBTree-Large), this gain is usually dwarfed by other overheads. Similar analysis at other thread levels continues to show these benefits, although increased bus contention and cache misses, as well as limited concurrency in some benchmarks, cause increased latency for all components of the timing breakdown.

HashTable: Since transactions are short (at most 2 objects read and 1 written) and conflicts are rare, the HashTable exhibits embarrassing parallelism: even RSTM is able to scale to a higher throughput than single-thread CGL. However, these properties prevent hardware from offering much additional benefit. The cost of copying is small, and since the read set is also small, the validation savings over RSTM are modest, resulting in about a $30 \%$ improvement.

RBTree: Tree rebalancing ensures that there are some conflicts in RBTree, while keeping the read and write sets above 10 and 3 objects, respectively. Validation is a significant overhead, and consequently ALoad enables RTM-F and RTM-Lite to double the performance of RSTM. All TMs demonstrate $\sim 10 \times$ speedup from 1 to 16 threads, and ALoad-assisted TMs are able to perform nearly twice as fast as CGL's peak despite starting off $\sim 5 \times$ slower at one thread.

RBTree-Large: RBTree-Large shows similar scaling behavior to RBTree, but RTM-F is able to leverage TLoad and TStore to eliminate long copy operations. RTM-Lite improves performance by $\sim 25 \%$ over RSTM by eliminating validation. RTM-F further improves performance by another $\sim 20 \%$ by reducing the copying overhead.

LFUCache: Due to the Zipf distribution used to choose which page to hit, there is virtually no concurrency in LFUCache, and all TMs flat-line as threads are added. Using ALoad allows RTM-F and RTM-Lite to outperform RSTM, but CGL's peak performance is still $\sim 3 \times$ higher.

LinkedList-Release: The use of early release keeps conflicts low, resulting in a high level of concurrency ( $\sim 10 \times$ speedup at 16 threads for all TMs). However, the high cost of metadata manipulation and bookkeeping (on average 64 objects are read and 62 released) keeps any TM from outperforming CGL's peak. At 16 threads, RTM and RTM-Lite achieve about $60 \%$ of CGL's single-thread performance. 
HashTable

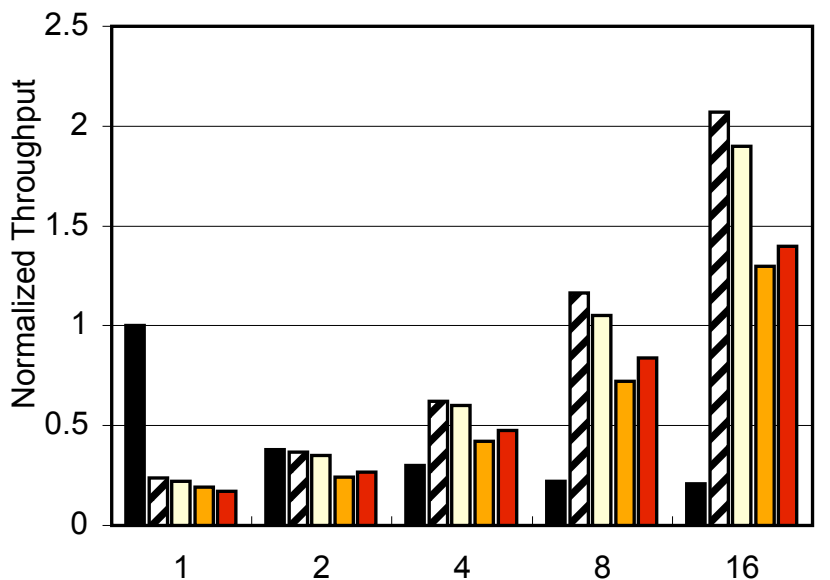

LinkedList-Release

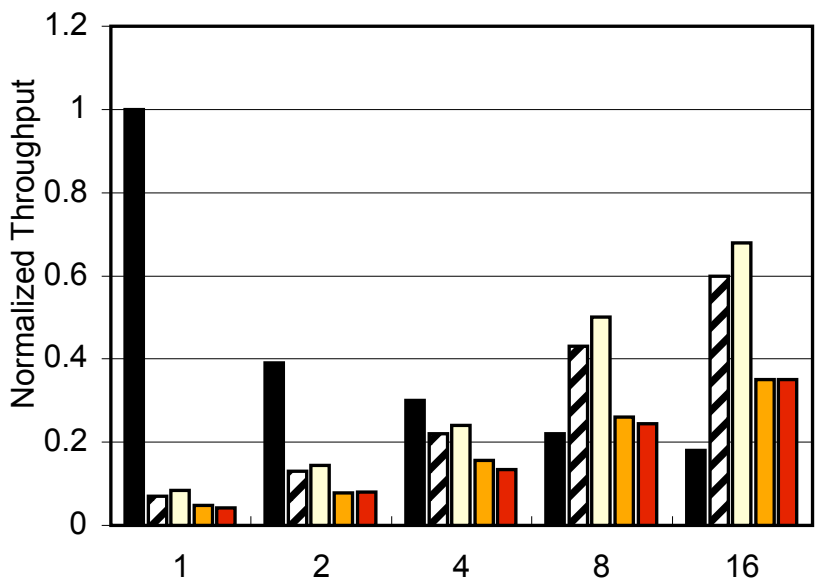

RBTree-Large

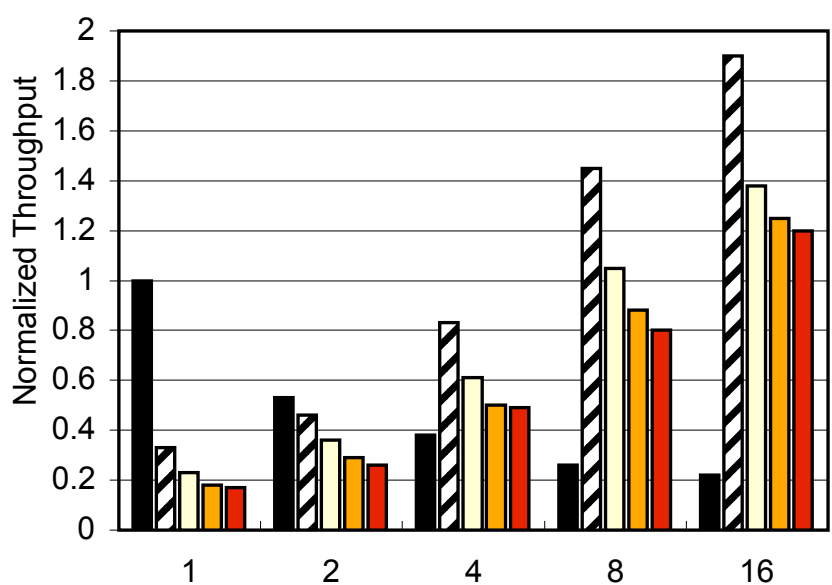

RBTree

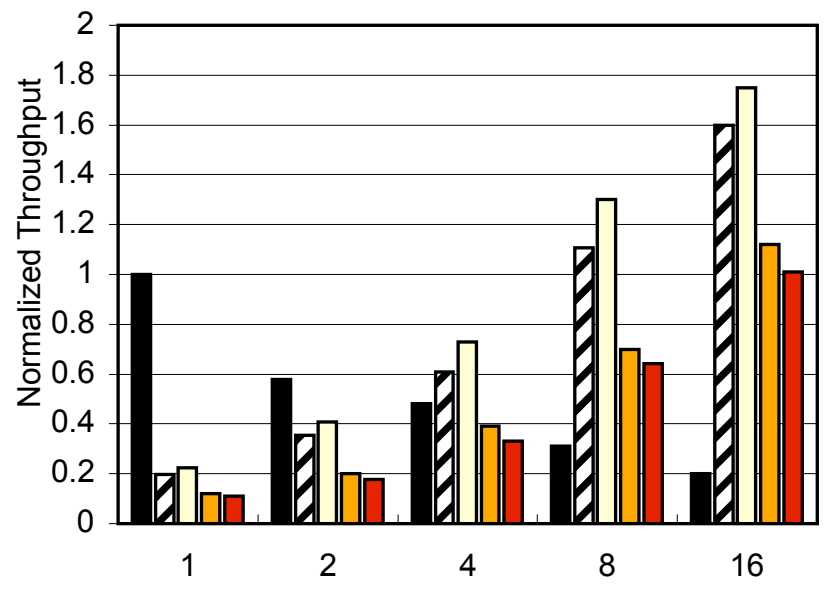

LFUCache

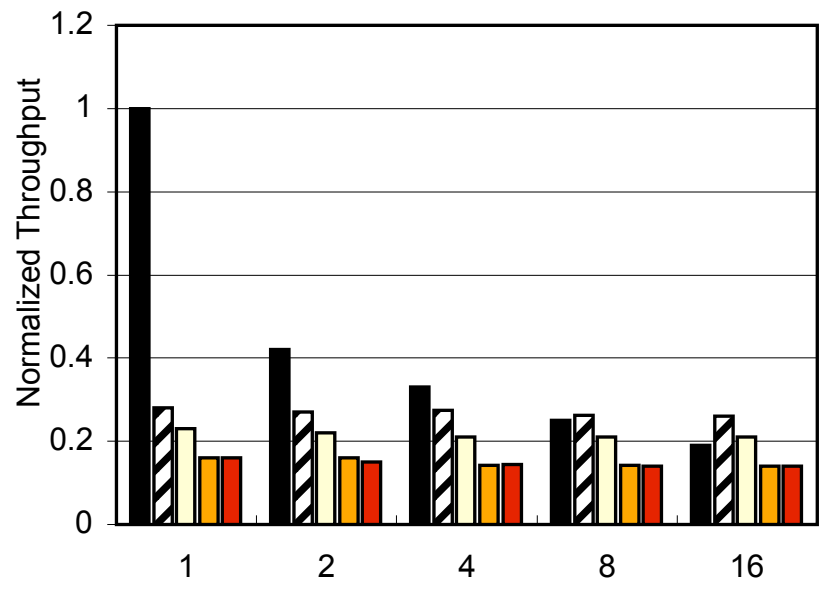

RandomGraph

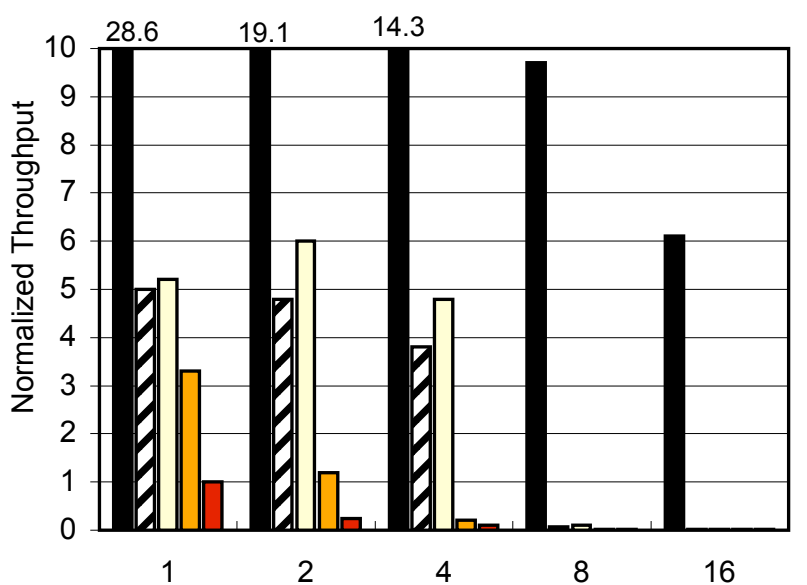

口CGL घRTM-F $\square R T M-L i t e \quad \square R T M-O \quad \square R S T M$

Figure 2: Throughput (transactions per second), normalized to single-thread CGL. RandomGraph is normalized to single-thread RSTM. 


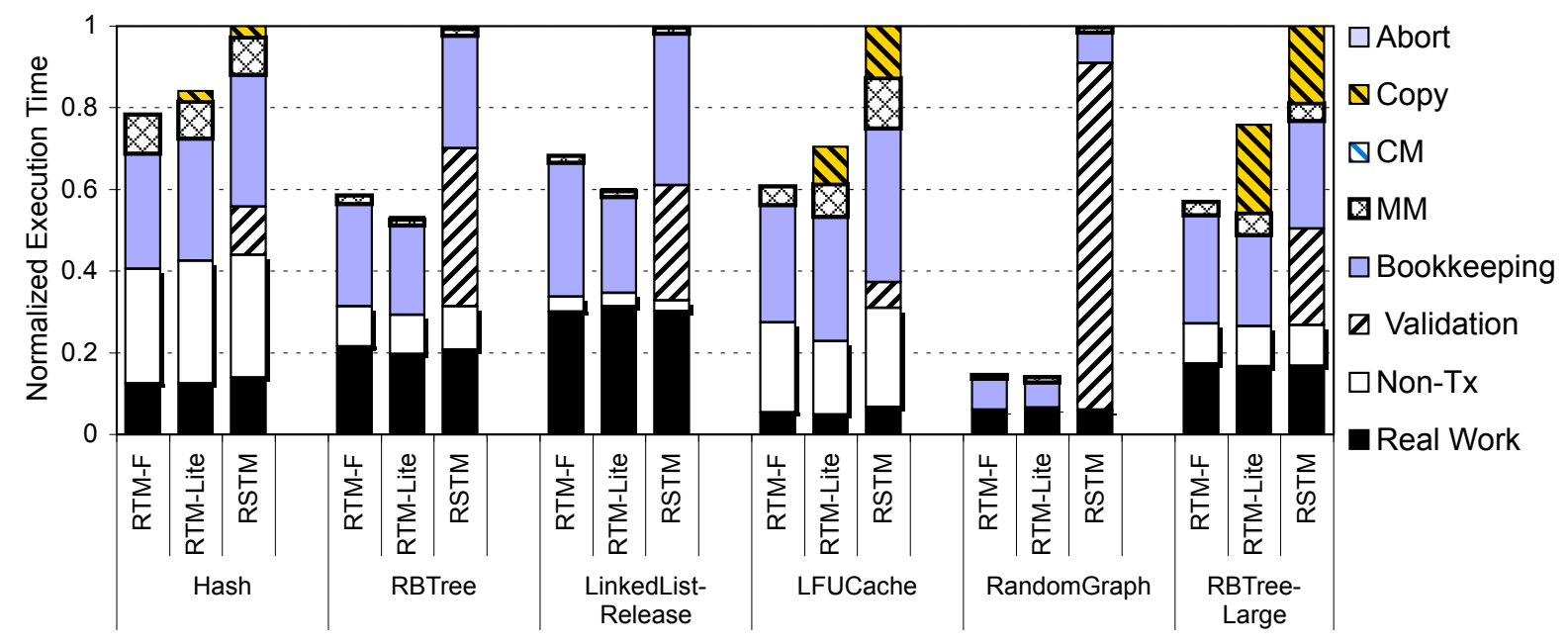

Figure 3: Breakdown of single-thread transaction latency, normalized to single-thread RSTM.

RandomGraph: Transactions in RandomGraph are complex; they read hundreds of objects, write tens of objects, and conflict with high probability. Validation is expensive and aborts are frequent. By leveraging ALoad, RTM-F and RTM-Lite can outperform RSTM by a factor of 5 at one thread. Similarly, RTM-O's use of a visible reader list enables it to avoid validation and dramatically outperform RSTM, although the two perform comparably when RSTM is configured to use visible reads. When there is any concurrency, however, the choice of eager acquire causes all TMs to livelock with the Polka contention manager. ${ }^{2}$

\subsection{Advantages of Policy Flexibility}

In order to evaluate the advantages of policy flexibility, we varied the conflict detection policy between eager and lazy. Figure 4 presents the results for HashTable, RBTree, LFUCache, and RandomGraph using 16 threads.

The HashTable and RBTree benchmarks demonstrate that in benchmarks that exhibit parallelism, the choice of conflict detection policy has a measurable effect. In HashTable, where conflicts are extremely rare, lazy acquire adds extra bookkeeping instructions without improving conflict resolution, resulting in a constant performance degradation that exceeds $15 \%$ for 16 threads. Similarly, RBTree suffers from the extra bookkeeping at low thread counts; at high thread counts, however, the use of lazy acquire enables RBTree to scale slightly better than eager acquire, ultimately achieving a $10 \%$ speedup.

As in RBTree, lazy transactions are slower than eager transactions in LFUCache at low thread levels due to extra bookkeeping. Since LFUCache admits no concurrency, eager acquire hurts performance at higher thread levels. As soon as an eager transaction calls open_RW on an object, it is vulnerable to conflicts with other threads. Since the likelihood of another thread trying to use the same object is high, increased concurrency decreases the likelihood of that transaction committing by a small factor. In contrast, lazy acquire actually improves performance. We expect that lazy acquire would not degrade due to contention; conflicts are only visible at the point where one transaction attempts to commit, and at that commit point conflicts are usually only realized between writers on the same object. Since the conflicting transactions are both about to commit, the likelihood of the conflict "winner" ultimately failing is low. The improvement in

\footnotetext{
${ }^{2}$ This livelocking behavior can be avoided by using a Greedy contention manager [6] modified to support invisible reads. Using Greedy, all TMs flat-line in RandomGraph as threads are added.
} 
HashTable

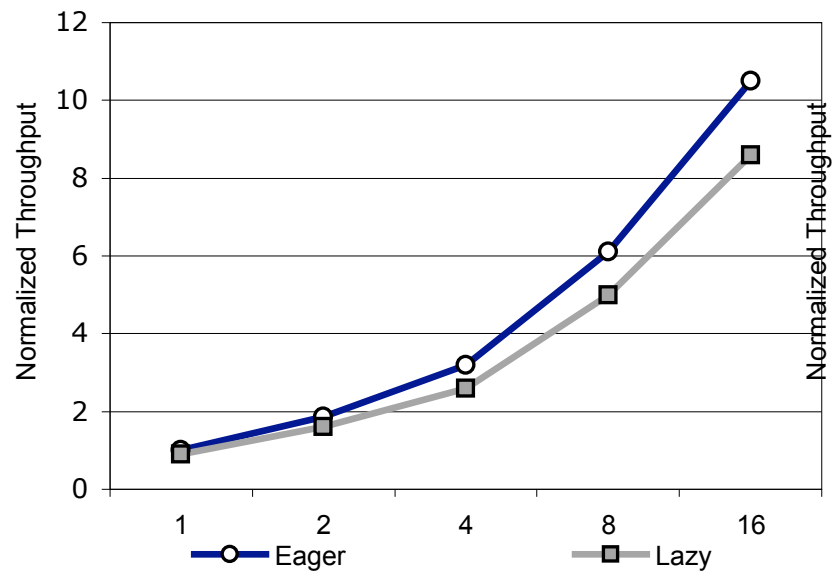

LFUCache

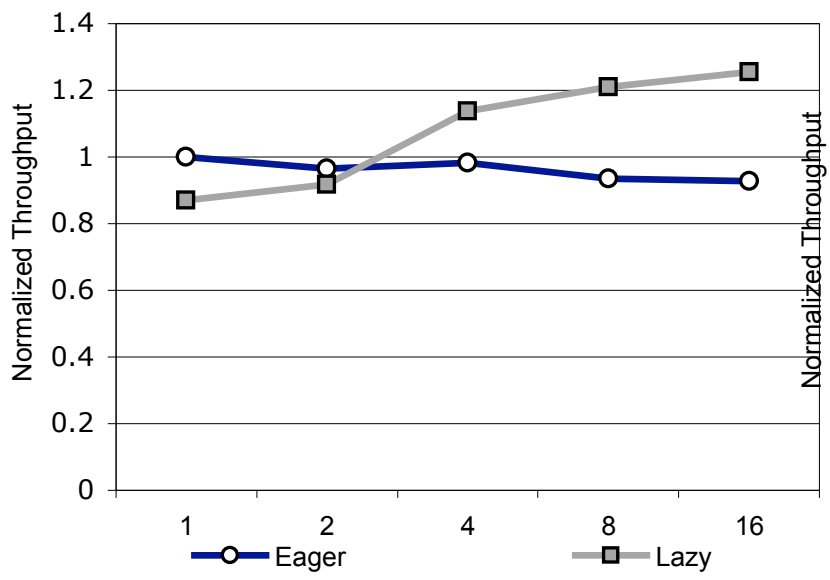

RBTree

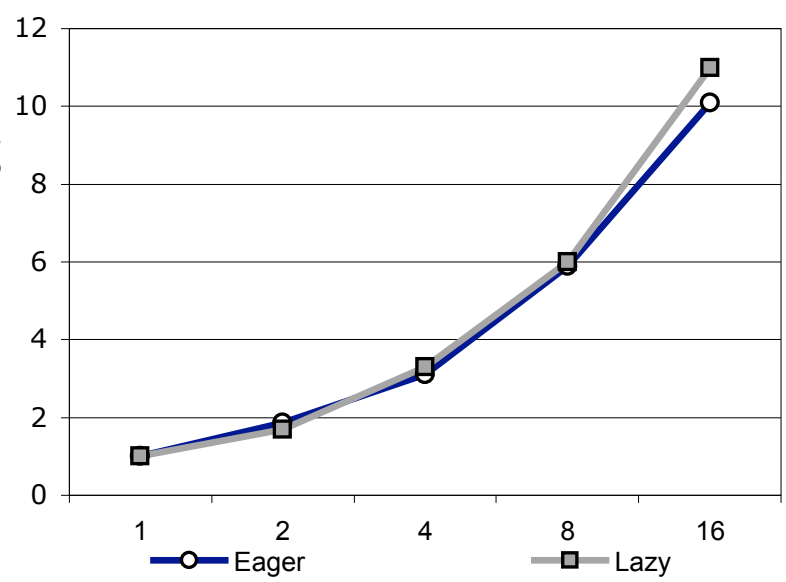

RandomGraph

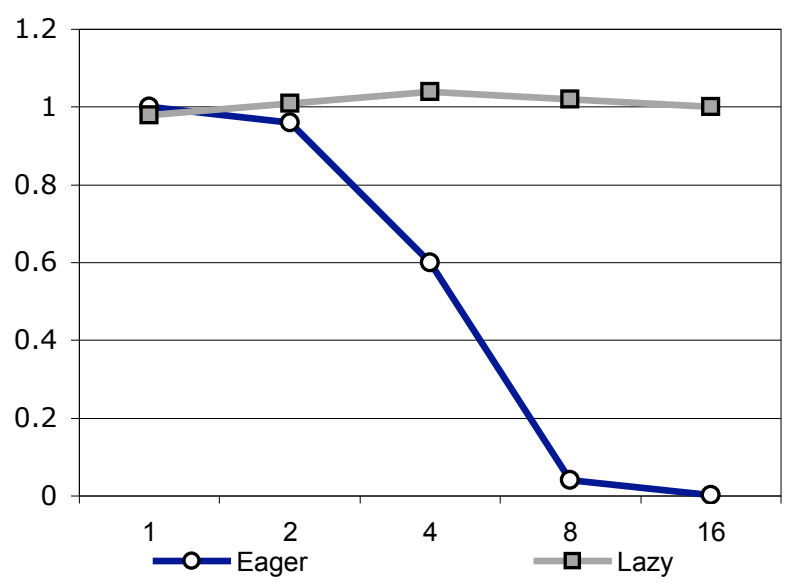

Figure 4: Eager vs. lazy conflict detection comparison, normalized to RTM Eager, 1 thread, as the number of threads is increased.

throughput with lazy acquire despite LFUCache's lack of concurrency is due to the ability to overlap one processor's transactional work with another processor's non-transactional work.

On RandomGraph, we had earlier noted that at high thread levels all TM systems livelocked under eager acquire. Figure 4 shows that lazy acquire avoids this pathological situation. RandomGraph transactions usually open_RW at least one highly contended object early, and then continue to read and write multiple objects. Since transactions run for tens of thousands of instructions, the likelihood of another transaction detecting and winning a conflict on the contended object is high under eager acquire. Unfortunately, that winner transaction is also likely to be aborted before reaching its commit point, for the same reason. With lazy acquire, this situation is avoided since conflicts are only detected at the very end of transactions. As in LFUCache, the winner of a conflict is unlikely to be aborted, unless it is by another transaction that is also about to commit. The consequences of this property are more dramatic than in LFUCache because the transactions are larger in space and time.

To summarize, even on a small set of benchmarks we find that neither lazy nor eager acquire is a silver bullet. Lazy demonstrates some desirable characteristics, and eager generally performs best at very low thread counts, but neither strategy consistently offers better performance at high thread levels. 


\subsection{Interaction of Hardware and Software Transactions}

We expect overflow transactions to occur rarely, with most transactions running in a short amount of time and fitting in the L1 cache. However, we must be sure that the occasional overflow transaction does not slow down fast-path transactions. Figure 5 reports a set of experiments designed to measure this effect.

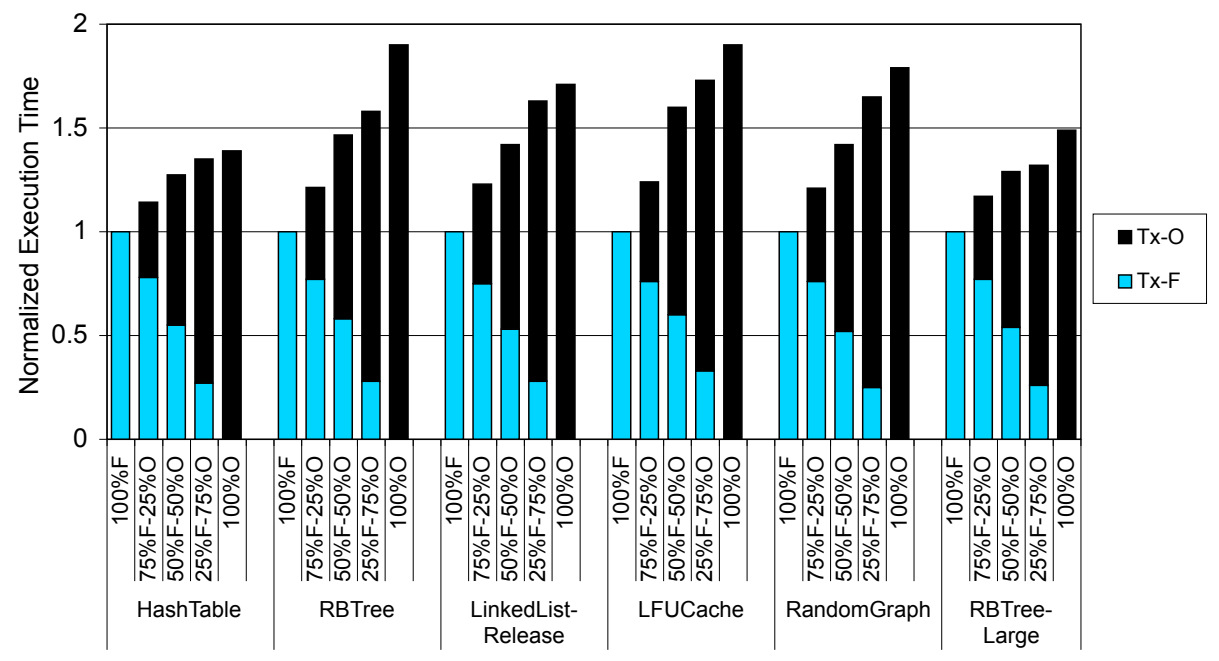

Figure 5: Breakdown of time spent in fast-path and in overflow mode, normalized to the all-fast-path execution (16 threads).

We run 16 threads all using lazy acquire. Each time a thread commits, it decides whether its next transaction should be run as overflow or fast-path using a uniform probability distribution. We vary the probability between 0 and 1 in increments of .25. Total execution time is measured, as well as the percentage of total execution time spent in fast-path transactions.

If we let $T_{f}$ be the time required to execute a fast-path transaction, and we let $T_{o}$ be the time required to execute an overflow transaction, then for a given probability $P_{o}$ that a thread runs as overflow, the total execution time should be $T_{f} \times\left(1-P_{o}\right)+T_{o} \times P_{o}$. In our experiments, we found that on average our results were within $4 \%$ of this predicted execution time, with the highest outlier only $7 \%$ above expected and the lowest outlier $6 \%$ below expected.

\section{Conclusions and Future Work}

We have described a transactional memory system, RTM, that uses a pair of hardware mechanisms to accelerate transactions managed by a software protocol: alert-on-update provides fast event-based communication for conflict detection; programmable data isolation allows a processor to hide speculative written lines from other processors and to continue to use speculatively read lines despite concurrent potentially conflicting writes on other processors.

Because it is based on a software TM system, RTM allows identical code to run efficiently on both legacy and updated hardware. Most hardware TM proposals, by contrast, assume a programming model that is difficult to implement efficiently on legacy machines. In particular, most hardware TM systems roll back everything on abort; most software TM systems distinguish between transactional data, which is rolled back, and nontransactional data, which is not. For the near term, this "mixed" programming model appears to provide an attractive migration path. 
In contrast to most previous proposals for transactional hardware, RTM supports both read-write and write-write sharing, allowing transactions to be highly optimistic when it is beneficial to be so. RTM's fast-path (fully hardware-supported) transactions require cache space only for speculative writes; lines that have only been read can safely be evicted. Transactions that nonetheless overflow hardware resources fall back gracefully to software, and interoperate smoothly with fast-path transactions. All transactions employ a software contention manager, enabling the use of adaptive or application-specific policies.

We have evaluated RTM using a detailed implementation in the GEMS/Simics simulation infrastructure [19]. For a suite of microbenchmarks with varying access patterns, we find that RTM outperforms RSTM by as much as a factor of two. The simpler RTM-Lite system, which relies on alert-on-update but not programmable data isolation, is effective at eliminating validation overhead, but loses to RTM for transactions that modify large objects. Echoing the findings of previous software TM studies, we find significant performance differences between eager and lazy conflict detection, with neither outperforming the other in all cases; this supports the need for policy flexibility.

In future work, we plan to explore a variety of topics, including performance sensitivity to processor organization parameters; simplified protocols (without transactional loads); implementations for other coherence protocols (e.g. MOESI or directory-based); additional hardware assists; nested transactions; gradual fall-back to software, with ongoing use of whatever fits in cache; other styles of RTM software (e.g., wordbased, blocking, and/or indirection-free); context identifiers for transactions implemented at a shared level of cache; and more realistic applications.

\section{References}

[1] C. S. Ananian, K. Asanovic, B. C. Kuszmaul, C. E. Leiserson, and S. Lie. Unbounded Transactional Memory. In Proc. of the 11th Intl. Symp. on High Performance Computer Architecture, pages 316-327, San Francisco, CA, Feb. 2005.

[2] E. D. Berger and B. G. Zorn. DieHard: Probabilistic Memory Safety for Unsafe Languages. In Proc. of the SIGPLAN 2006 Conf. on Programming Language Design and Implementation, Ottawa, ON, Canada, June 2006.

[3] W. Chuang, S. Narayanasamy, G. Venkatesh, J. Sampson, M. V. Biesbrouck, G. Pokam, B. Calder, and O. Colavin. Unbounded Page-Based Transactional Memory. In Proc. of the 12th Intl. Conf. on Architectural Support for Programming Languages and Operating Systems, pages 347-358, San Jose, CA, Oct. 2006.

[4] P. Damron, A. Fedorova, Y. Lev, V. Luchangco, M. Moir, and D. Nussbaum. Hybrid Transactional Memory. In Proc. of the 12th Intl. Conf. on Architectural Support for Programming Languages and Operating Systems, San Jose, CA, Oct. 2006.

[5] K. Fraser and T. Harris. Concurrent Programming Without Locks. Submitted for publication, 2004. Available as research.microsoft.com/ tharris/drafts/cpwl- submission.pdf.

[6] R. Guerraoui, M. Herlihy, and B. Pochon. Polymorphic Contention Management in SXM. In Proc. of the 19th Intl. Symp. on Distributed Computing, Cracow, Poland, Sept. 2005.

[7] L. Hammond, B. D. Carlstrom, V. Wong, B. Hertzberg, M. Chen, C. Kozyrakis, and K. Olukotun. Programming with Transactional Coherence and Consistency (TCC). In Proc. of the 11th Intl. Conf. on Architectural Support for Programming Languages and Operating Systems, pages 1-13, Boston, MA, Oct. 2004.

[8] L. Hammond, V. Wong, M. Chen, B. Hertzberg, B. Carlstrom, M. Prabhu, H. Wijaya, C. Kozyrakis, and K. Olukotun. Transactional Memory Coherence and Consistency. In Proc. of the 31st Intl. Symp. on Computer Architecture, München, Germany, June 2004.

[9] M. Herlihy, V. Luchangco, M. Moir, and W. N. Scherer III. Software Transactional Memory for Dynamic-sized Data Structures. In Proc. of the 22nd ACM Symp. on Principles of Distributed Computing, pages 92-101, Boston, MA, July 2003.

[10] M. Herlihy and J. E. Moss. Transactional Memory: Architectural Support for Lock-Free Data Structures. In Proc. of the 20th Intl. Symp. on Computer Architecture, pages 289-300, San Diego, CA, May 1993. Expanded version available as CRL 92/07, DEC Cambridge Research Laboratory, Dec. 1992. 
[11] Intel Corporation. Intel Itanium Architecture Software Developer's Manual. Revision 2.2, Jan. 2006.

[12] R. Kalla, B. Sinharoy, and J. M. Tendler. IBM Power5 Chip: A Dual-core Multithreaded Processor. IEEE Micro, 24(2):40-47, Mar.-Apr. 2004.

[13] P. Kongetira, K. Aingaran, and K. Olukotun. Niagara: A 32-Way Multithreaded SPARC Processor. In IEEE Micro, pages 21-29, Mar.-Apr. 2005.

[14] S. Kumar, M. Chu, C. J. Hughes, P. Kundu, and A. Nguyen. Hybrid Transactional Memory. In Proc. of the 11th ACM Symp. on Principles and Practice of Parallel Programming, New York, NY, Mar. 2006.

[15] S. Lu, J. Tucek, F. Qin, and Y. Zhou. AVIO: Detecting Atomicity Violations via Access Interleaving Invariants. In Proc. of the 12th Intl. Conf. on Architectural Support for Programming Languages and Operating Systems, San Jose, CA, Oct. 2006.

[16] V. J. Marathe, W. N. Scherer III, and M. L. Scott. Adaptive Software Transactional Memory. In Proc. of the 19th Intl. Symp. on Distributed Computing, Cracow, Poland, Sept. 2005.

[17] V. J. Marathe, M. F. Spear, C. Heriot, A. Acharya, D. Eisenstat, W. N. Scherer III, and M. L. Scott. Lowering the Overhead of Software Transactional Memory. In ACM SIGPLAN Workshop on Languages, Compilers, and Hardware Support for Transactional Computing, Ottawa, ON, Canada, June 2006. Held in conjunction with PLDI 2006. Expanded version available as TR 893, Dept. of Computer Science, Univ. of Rochester, Mar. 2006.

[18] J. F. Martínez and J. Torrellas. Speculative Synchronization: Applying Thread-Level Speculation to Explicitly Parallel Applications. In Proc. of the 10th Intl. Conf. on Architectural Support for Programming Languages and Operating Systems, pages 18-29, San Jose, CA, Oct. 2002.

[19] M. M. K. Martin, D. J. Sorin, B. M. Beckmann, M. R. Marty, M. Xu, A. R. Alameldeen, K. E. Moore, M. D. Hill, and D. A. Wood. Multifacet's General Execution-driven Multiprocessor Simulator (GEMS) Toolset. In ACM SIGARCH Computer Architecture News, Sept. 2005.

[20] P. E. McKenney, J. Appavoo, A. Kleen, O. Krieger, R. Russel, D. Sarma, and M. Soni. Read-Copy Update. In Proc. of the Ottawa Linux Symp., July 2001.

[21] C. McNairy and R. Bhatia. Montecito: A Dual-core, Dual-thread Itanium Processor. IEEE Micro, 25(2):10-20, Mar.-Apr. 2005.

[22] M. M. Michael. Hazard Pointers: Safe Memory Reclamation for Lock-Free Objects. IEEE Trans. on Parallel and Distributed Systems, 15(8), Aug. 2004.

[23] K. E. Moore, J. Bobba, M. J. Moravan, M. D. Hill, and D. A. Wood. LogTM: Log-based Transactional Memory. In Proc. of the 12th Intl. Symp. on High Performance Computer Architecture, Austin, TX, Feb. 2006.

[24] J. E. B. Moss. Open Nested Transactions: Semantics and Support. In Proc. of the 4th IEEE Workshop on Memory Performance Issues, Austin, Texas, Feb. 2006. Held in conjunction with HPCA 2006.

[25] J. Oplinger and M. S. Lam. Enhancing Software Reliability with Speculative Threads. In Proc. of the 10th Intl. Conf. on Architectural Support for Programming Languages and Operating Systems, pages 184-196, San Jose, CA, Oct. 2002.

[26] R. Rajwar and J. R. Goodman. Speculative Lock Elision: Enabling Highly Concurrent Multithreaded Execution. In Proc. of the 34th Intl. Symp. on Microarchitecture, Austin, TX, Dec. 2001.

[27] R. Rajwar and J. R. Goodman. Transactional Lock-Free Execution of Lock-Based Programs. In Proc. of the 10th Intl. Conf. on Architectural Support for Programming Languages and Operating Systems, pages 5-17, San Jose, CA, Oct. 2002.

[28] R. Rajwar, M. Herlihy, and K. Lai. Virtualizing Transactional Memory. In Proc. of the 32nd Intl. Symp. on Computer Architecture, Madison, WI, June 2005.

[29] B. Saha, A.-R. Adl-Tabatabai, R. L. Hudson, C. C. Minh, and B. Hertzberg. McRT-STM: A High Performance Software Transactional Memory System for a Multi-Core Runtime. In Proc. of the 11th ACM Symp. on Principles and Practice of Parallel Programming, New York, NY, Mar. 2006.

[30] W. N. Scherer III and M. L. Scott. Advanced Contention Management for Dynamic Software Transactional Memory. In Proc. of the 24th ACM Symp. on Principles of Distributed Computing, Las Vegas, NV, July 2005.

[31] N. Shavit and D. Touitou. Software Transactional Memory. Distributed Computing, 10(2):99-116, Feb. 1997. Originally presented at the 14th ACM Symp. on Principles of Distributed Computing, Aug. 1995. 
[32] A. Shriraman, V. J. Marathe, S. Dwarkadas, M. L. Scott, D. Eisenstat, C. Heriot, W. N. Scherer III, and M. F. Spear. Hardware Acceleration of Software Transactional Memory. In ACM SIGPLAN Workshop on Languages, Compilers, and Hardware Support for Transactional Computing, Ottawa, ON, Canada, June 2006. Held in conjunction with PLDI 2006. Expanded version available as TR 887, Dept. of Computer Science, Univ. of Rochester, Dec. 2005, revised Mar. 2006.

[33] M. F. Spear, V. J. Marathe, W. N. Scherer III, and M. L. Scott. Conflict Detection and Validation Strategies for Software Transactional Memory. In Proc. of the 20th Intl. Symp. on Distributed Computing, Stockholm, Sweden, Sept. 2006.

[34] J. Steffan and T. Mowry. The Potential for Using Thread-Level Data Speculation to Facilitate Automatic Parallelization. In Proc. of the 4th Intl. Symp. on High Performance Computer Architecture, pages 2-13, Las Vegas, NV, Feb. 1998.

[35] T. von Eicken, D. E. Culler, S. C. Goldstein, and K. E. Schauser. Active Messages: A Mechanism for Integrated Communication and Computation. In Proc. of the 19th Intl. Symp. on Computer Architecture, pages 256-266, Gold Coast, Australia, May 1992.

[36] C. Zilles and L. Baugh. Extending Hardware Transactional Memory to Support Non-Busy Waiting and NonTransactional Actions. In ACM SIGPLAN Workshop on Languages, Compilers, and Hardware Support for Transactional Computing, Ottawa, ON, Canada, June 2006. Held in conjunction with PLDI 2006. 


\section{A TMESI Protocol Transitions}

Figure 6 contains the transition diagram for the stable states of the TMESI protocol, with transactional tags for $T M, T E$, and $T S$ states. Orthogonal A bits are not shown.

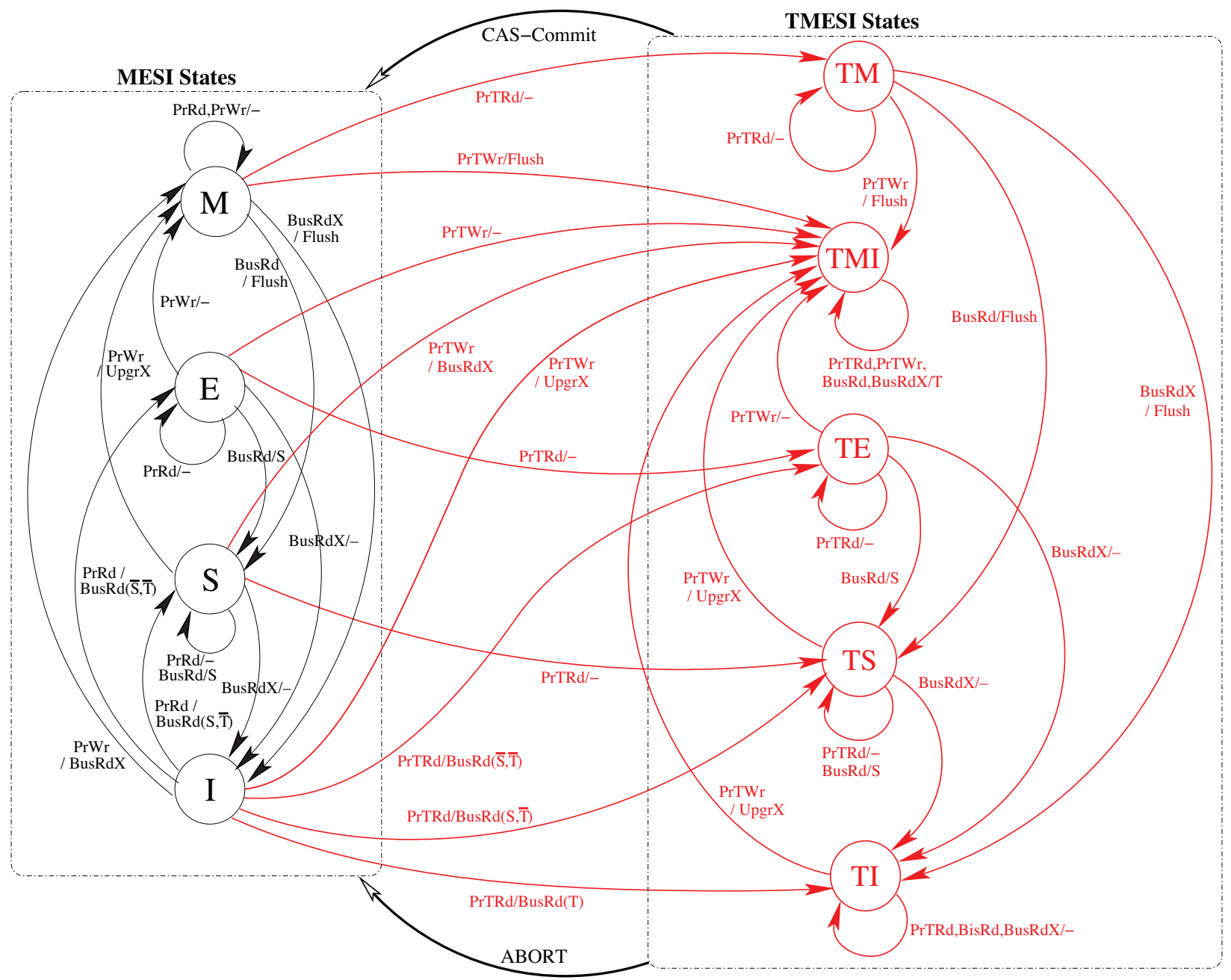

Figure 6: TMESI Protocol.

Dashed boxes enclose the MESI and TMESI subsets of the state space. In the wake of a CAS-Commit, $T M, T E, T S$, and $T I$ revert to $M, E, S$, and $I$, respectively; TMI reverts to $M$ if the CAS-Commit succeeds, or to $I$ if it fails. Notation on transitions is conventional: the part before the slash is the triggering message; after is the ancillary action ("-' means none). "Flush" indicates that the cache writes the line out to the bus. S and T indicate signals on the "shared" and "threatened" bus lines respectively. Plain, they indicate assertion by the local processor; parenthesized, they indicate the signals that accompany the response to a BusRd request. An overbar means "not signaled". For simplicity, we assume that the base protocol prefers memory-cache transfers over cache-cache transfers. In all cases, a cache responds to Upgr (upgrade to $M$ ) requests the same way it responds to BusRdX messages. 


\section{B Simplified TMESI Protocol}

Figure 7 contains the transition diagram for the stable states of the simplified TMESI protocol alluded to in Section 2.3. By omitting the transactional tags for $T M, T E$, and $T S$ states, we obtain a significantly simpler protocol, at the cost of reloading lines that are read twice in the same transaction, and are threatened (for the first time) in between. As in Figure 6, orthogonal A bits are not shown.

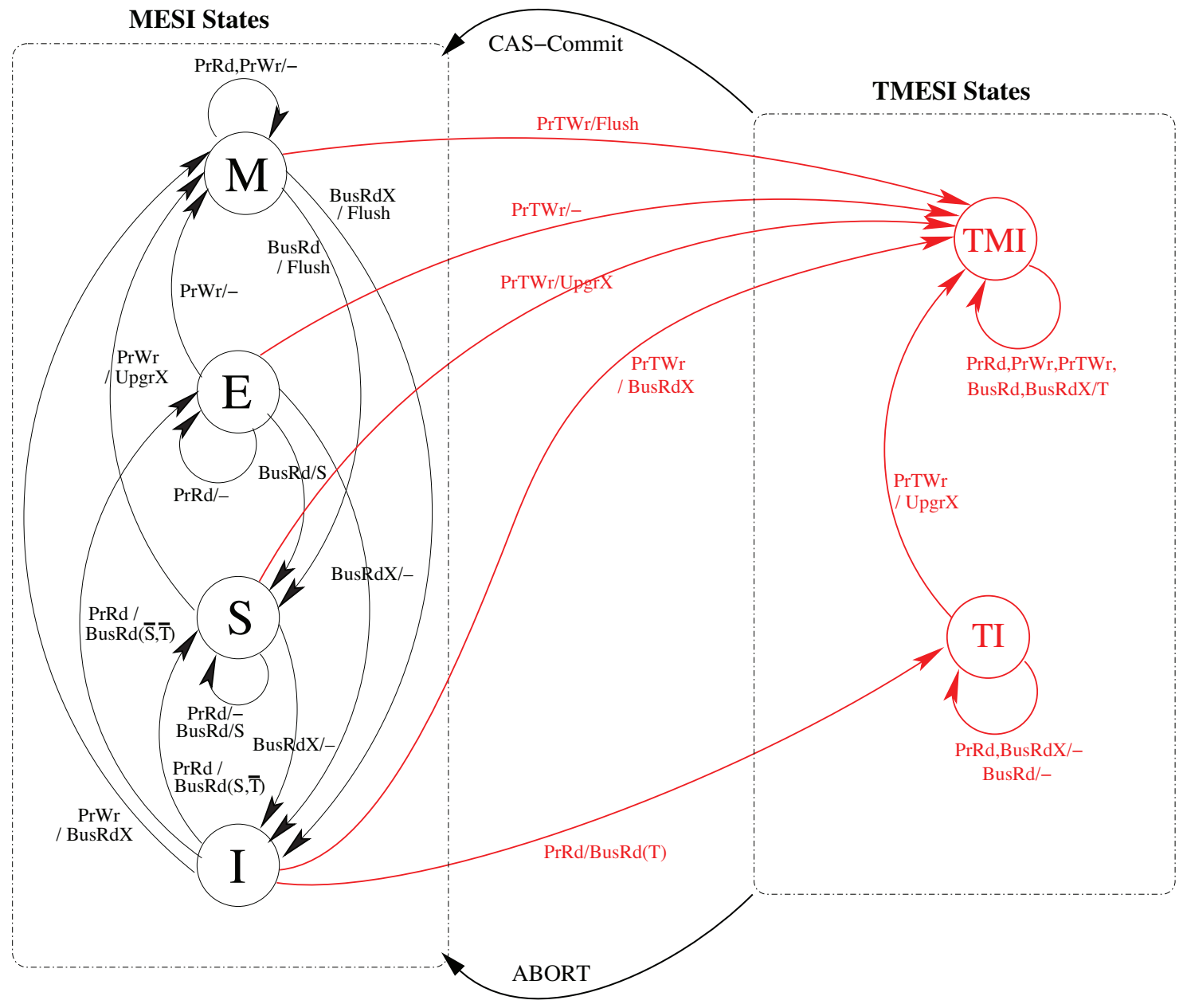

Figure 7: Simplified TMESI Protocol.

\section{Example}

Figure 8 illustrates the interactions among three simple concurrent transactions, with the interleaving of accesses with lazy acquire at the top and cache tag arrays at various points in time at the bottom. OH (X) indicates the header of any object $x$. Only the transactional instructions are shown. Numbers indicate the order in which instructions occur. At the beginning of each transaction, RTM software executes a SetHandler instruction, initializes a transaction descriptor (in software), and ALoads that descriptor. Though the open calls are not shown explicitly, RTM software also executes an ALoad on each object header at the time of the open and before the initial TLoad or TStore. We use AM, AE, and AS to indicate MESI states with the A bit set. 


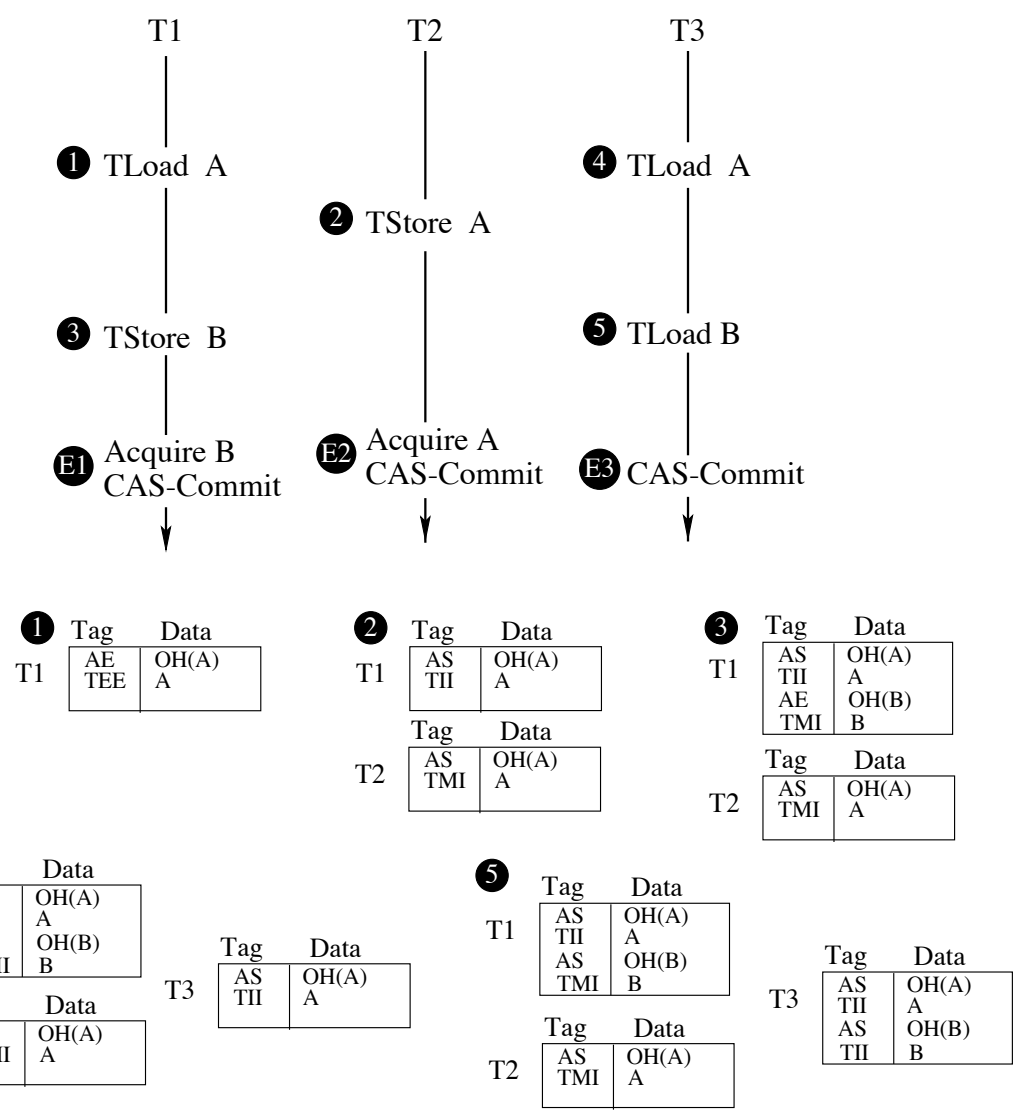

Figure 8: Execution of Transactions. Top: interleaving of accesses in three transactions, with lazy acquire. Bottom: Cache tag arrays at various event points. $(\mathrm{OH}(\mathrm{x})$ is used to indicate the header of object $\mathrm{x}$.)

Let us assume that initially objects A and B are invalid in all caches. At (1) transaction T1 performs a TLoad of object A. RTM software will have ALoaded A's header into T1's cache in state AE (since it is the only cached copy) at the time of the open. The referenced line of A is then loaded in $T E$. When the store happens in $\mathrm{T} 2$ at 2 , the line in TE in $\mathrm{T} 1$ sees a BusRdX message and drops to $T I$. The line remains valid, however, and $\mathrm{T} 1$ can continue to use it until T2 acquires A (thereby aborting T1) or T1 itself commits. Regardless of T1's outcome, the $T I$ line must drop to $I$ to reflect the possibility that a transaction threatening that line can subsequently commit.

At 3 T1 performs a TStore to object B. RTM loads B's header in state $A E$ at the time of the open, and $\mathrm{B}$ itself is loaded in $T M I$, since the write is speculative. If $\mathrm{T} 1$ commits, the line will revert to $M$, making the TStore's change permanent. If $\mathrm{T} 1$ aborts, the line will revert to $I$, since the speculative value will at that point be invalid.

At 4 transaction T3 performs a TLoad on object A. Since T2 holds the line in TMI, it asserts the T signal in response to T3's BusRd message. This causes T3 to load the line in TI, giving it access only until it commits or aborts (at which point it loses the protection of software conflict detection). Prior to the TLoad, RTM software will have ALoaded A's header into T3's cache during the open, causing T2 to assert the S signal and to drop its own copy of the header to AS. If T2 acquires A while T3 is active, its BusRdX on A's header will cause an invalidation in T3's cache and thus an immediate abort of T3.

Event 5 is similar to 4, and B is also loaded in $T I$. 
We now consider the ordering of events E1, E2, and E3.

1. E1 happens before E2 and E3: When T1 acquires B's header, it invalidates the line in T3's cache. This causes T3 to abort. T2, however, can commit. When it retries, T3 will see the new value of A from T1's commit.

2. E2 happens before E1 and E3: When T2 acquires A's header, it aborts both T1 and T3.

3. E3 happens before E1 and E2: Since T3 is only a reader of objects, and has not been invalidated by writer acquires, it commits. T2 can similarly commit, if E1 happens before E2, since T1 is a reader of A. Thus, the ordering E3, E1, E2 will allow all three transactions to commit. TCC [8] would also admit this scenario, but to the best of our knowledge no other hardware or hybrid TM scheme would do so, because of eager conflict detection. RTM enforces consistency with a single BusRdX per object header. In contrast, TCC must broadcast all speculatively modified lines at commit time.

\section{Nontransactional Use of Alert-on-Update}

As noted in Section 1, alert-on-update could be used for any task that benefits from fine-grain access control. We enumerate several examples here.

To maximize its flexibility, we propose two variants of the ALoad instruction: The first, as described in Section 2.1, generates an alert when the line is evicted from the cache. The second also generates an alert if the line is written or, optionally, read locally.

\section{D.1 Fast User-space Mutexes}

The low latency of alert signals shifts the tradeoff between spinning and yielding on lock acquisition failure, especially in the case of user-level thread packages. Ideally, a thread $T$ would yield immediately when it fails to acquire lock $L$, and would wake immediately when $L$ is released. To approximate this behavior, we need only prefix the acquire attempt with an ALoad of the lock. Then, on lock failure, $T$ can yield without ARelease-ing the line. On a subsequent alert the handler would switch back to $T$. In this manner no cycles are wasted spinning on an unavailable lock, and no bus traffic is generated by multiple unsuccessful acquire attempts.

For optimal performance, the thread package may specify that the alert handler attempts to acquire $L$ on $T$ 's behalf when an alert is given. This ensures the lowest latency between the release of $L$ and its acquisition by $T$. Additionally, if $L$ is acquired by $T^{\prime}$ before the alert handler can acquire it for $T$, the thread switch to $T$ can be avoided. Furthermore, using the variant of ALoad that also generates alerts for local writes, this method is appropriate regardless of whether the lock holder and failing acquirer reside on the same processor or separate processors. This technique is useful both with and without transactions, and thus is more general (and carries less overhead due to transaction rollback) than a similar proposal by Zilles and Baugh [36].

\section{D.2 Asynchronous Message Handling}

Communication mechanisms (e.g., Active Messages [35]) in which messages are received asynchronously generally require either user-level polling or remote interrupts and operating system intervention, both of which are expensive. By ALoading a "doorbell" location, a thread can provide its message-passing peers with a simple, efficient way to notify it when a message buffer has been filled and is ready for receipt. 


\section{D.3 Fast Rollback in Nonblocking Algorithms}

Many nonblocking algorithms have distinct phases: first a shared location is read, then a new value is computed for the shared location, and finally the thread attempts to CAS the new value in place of the old value. When the value of the shared location changes during the middle of the computation phase, the computing thread is doomed to abort, but cannot detect its fate without continuous polling.

To avoid both polling and wasted work, a thread might ALoad the shared location, and register an alert handler that restarts the operation immediately. Using this idiom, if the location changes the thread will immediately discard its wasted effort and return to the phase in which it reads the shared location. Depending on the complexity of the computation in the second phase, the alert handler may wish to throw an exception, issue a longjmp(), or simply execute an unconditional branch.

This technique is applicable not only to nonblocking algorithms, but also to read-copy-update algorithms [20] and many software TM systems. It is likely that rollback will entail more than a simple jump instruction for these applications, since they are likely to allocate deep data structures during their computation phase.

\section{D.4 Hazard Pointers}

Compare-and-swap instructions are susceptible to the "ABA" problem, in which thread $T_{1}$ reads $L==A$ and then CASes $L$ to $Z$, but in the meantime two other CASes are issued by other threads, changing $L$ from $A$ to $B$ and back to $A$ again. In algorithms that require that $L$ remain constant from the first read by $T_{1}$ to the final CAS, more complicated measures such as hazard pointers have been proposed [22].

We observe, however, that the fast rollback technique of the previous subsection solves the ABA problem implicitly. Any change to $L$ will result in an alert to $T_{1}$, and the absence of any alert between the initial read of $L$ and the moment when $T_{1}$ CASes $L$ from $A$ to $Z$ indicates that there was no ABA risk.

Depending on the implementation of CAS, this technique might result in unnecessary alerts. In particular, it is common for the CAS microcode to begin with a get-exclusive bus message. Thus if $L$ is not $A$ when

$T_{1}$ tries to execute CAS ( \& L, A, Z), the CAS will still cause remote processors to take an alert on $L$. For highly contended locations, we suspect that a software test-and-CAS operation, which loads $L$, compares $L$ to $A$, and conditionally issues a hardware CAS only if $L==A$ would effectively mitigate this risk without requiring an additional hardware instruction.

\section{D.5 Debugging}

Modern microprocessors currently provide limited support for debuggers through watchpoint registers. On the $\mathrm{x} 86$, for example, there are 4 debug registers which may be used to monitor memory regions of 1,2 or 4 contiguous bytes. With pervasive parallelism, four debug watchpoints registers may not be enough. Alerton-update (the variant that also generates alerts on local writes) allows the debugger to set watchpoints at only coarser cache-line granularity but supports a larger number of watchpoints, up to the size of the cache.

More recently Lu et al. [15] describe "AVIO", a statistical invariant defining technique used to detect atomicity violations. They explore hardware support for the AVIO system, which tracks the preceding access instruction type and remote downgrade requests. It is conceivable that alert-on-update could support AVIO type techniques. Alert-on-update implicitly indicates that the most recent access was either a local $\mathrm{read} /$ write or a remote read. AVIO also tracks in hardware the most recent downgrade request. With AOU these would be tracked in software by the alert handler. 


\section{D.6 Code Security}

Due to the fine (cache line) granularity of the alert-on-update mechanism, it is suitable for detecting and reacting to memory corruption in settings where page-based detection mechanisms are either too expensive or too space-inefficient.

Buffer Overflows In order to detect buffer overflows in legacy code, a program could ALoad portions of its stack. A particularly appealing technique, inspired by DieHard [2], is to use randomization across installations of an application: the compiler could choose a random number of cache lines of buffering between stack frames at compile time, and then ALoad those empty lines as part of the function prologue. Since the size of the padding is known at compile time, parameter passing via the stack would not be compromised, but the randomization of the padding across builds of the program would increase the likelihood that an attacker could not attack multiple installations of a program with the same input. To do so would very likely result in an alert-based detection for at least one installation, thereby revealing the buffer vulnerability.

Read-Only Fields and Methods At compile time, one might order the fields of a class so that all const member fields are adjacent. Then the object constructor could ALoad that line immediately after initialization. In this manner, software could quickly detect when memory safety is violated. In a similar manner, if a method is const, then it would be possible in the function prologue to ALoad the lines corresponding to the entire class's representation in memory, to detect when member functions discard const pointers.

Notification on Dynamic Code Modification Another appealing use of ALoad is to permit finegrained protection of code pages. Although the majority of applications do not require the ability to dynamically modify their code, and are well served by using page-level protection, there is no mechanism by which applications that modify their own code pages can ensure that malicious code does not make unauthorized modifications.

With ALoad however, a program could set the alert bits of code pages, and then use a private signature field to indicate to the alert handler when the application is making a safe modification to its code. If the alert handler is invoked and the private signature matches a hash of the address causing the alert, the handler can safely assume that the alert was caused by a trusted operation. If the alert handler detects a signature conflict, it can assume that the code pages are being modified by an untrusted agent, and can raise an appropriate exception.

\section{E Nontransactional Use of Programmable Data Isolation}

Like Alert-on-Update, Programmable Data Isolation can be used for purposes other than transactional memory, though the opportunities do not appear to be as extensive as those listed in the previous section. As noted by designers of hardware TM systems (e.g., Hammond et al. [7], possilibites include (1) ordered, threadlevel speculation [34], and (2) speculative execution in sequential programs for reliability [25] or exception rollback. The chief difference here between previous implementations and one based on programmable data isolation would be the option, with the latter, of rolling back a selected subset of a program's data, leaving updates of other data visible. 